\title{
Use-Cases des autonomen Fahrens
}

\author{
Walther Wachenfeld, Hermann Winner, Chris Gerdes, Barbara Lenz, \\ Markus Maurer, Sven A. Beiker, Eva Fraedrich, Thomas Winkle
}

\footnotetext{
W. Wachenfeld $(\square)$

Technische Universität Darmstadt, Fachgebiet Fahrzeugtechnik - FZD, Deutschland wachenfeld@fzd.tu-darmstadt.de

H. Winner

Technische Universität Darmstadt, Fachgebiet Fahrzeugtechnik - FZD, Deutschland winner@fzd.tu-darmstadt.de
}

J.C. Gerdes

Stanford University, Dept. of Mechanical Engineering Center for Automotive Research at Stanford, USA

gerdes@stanford.edu/gerdes@cdr.stanford.edu

B. Lenz

Deutsches Zentrum für Luft- und Raumfahrt e.V, Institut für Verkehrsforschung, Deutschland Barbara.Lenz@dlr.de

M. Maurer

TU Braunschweig, Institut für Regelungstechnik, Deutschland maurer@ifr.ing.tu-bs.de

S. A. Beiker

formerly Stanford University, Center for Automotive Research at Stanford sven@svenbeiker.com

E. Fraedrich

Humboldt-Universität zu Berlin, Geographisches Institut, Deutschland eva.fraedrich@geo.hu-berlin.de

T. Winkle

Technische Universität München - TUM, Maschinenwesen, Lehrstuhl für Ergonomie, Deutschland winkle@carforensic.com 


\section{Inhaltsverzeichnis}

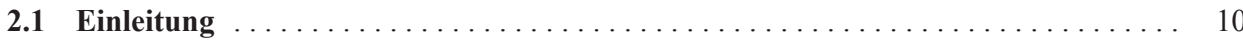

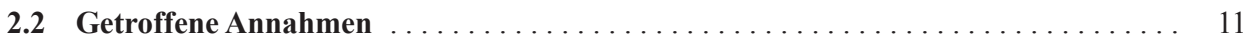

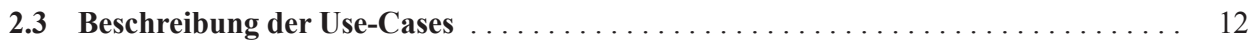

2.3.1 Autobahnautomat mit Verfügbarkeitsfahrer-Autobahnpilot ............. 12

2.3.2 Autonomes Valet-Parken .............................. 14

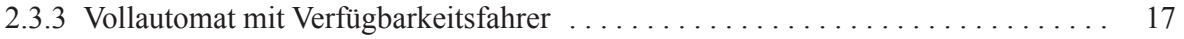

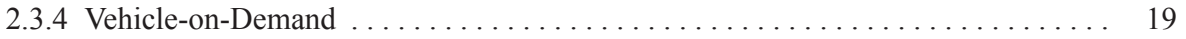

2.4 Ausgewählte Merkmale zur Beschreibung der Use-Cases $\ldots \ldots \ldots \ldots \ldots \ldots \ldots \quad 21$

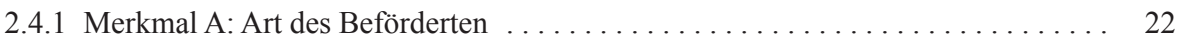

2.4.2 Merkmal B: Maximal zulässige Gesamtmasse $\ldots \ldots \ldots \ldots \ldots \ldots \ldots \ldots \ldots . \ldots \ldots$

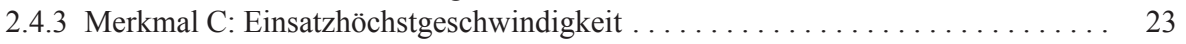

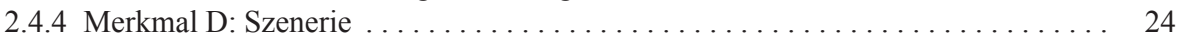

2.4.5 Merkmal E: Dynamische Elemente ..................... 26

2.4.6 Merkmal F: Informationsfluss zwischen Fahrroboter und anderen Instanzen . . . . 27

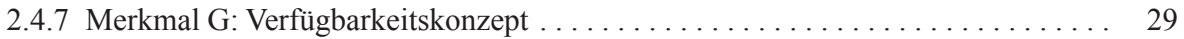

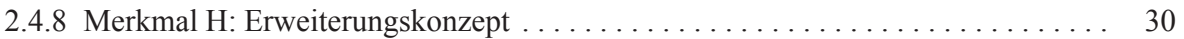

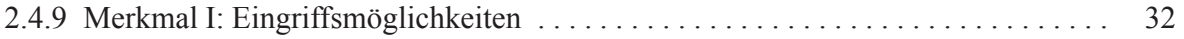

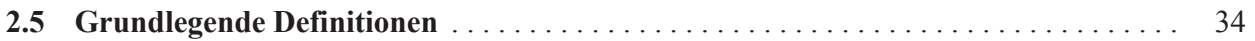

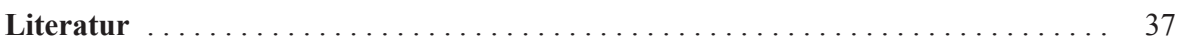

\subsection{Einleitung}

Der Begriff des autonomen Fahrens wurde mithilfe der technischen Definition des Automatisierungsgrads ,vollautomatisiert“ nach BASt [1] und zusätzlich durch das Verständnis der „Selbstbestimmung im Rahmen eines übergeordneten (Sitten)-Gesetzes“ “2] in Kap. 1 beschrieben. Trotzdem kann innerhalb dieser Rahmendefinition eine Vielfalt an Einsatzmöglichkeiten und Ausprägungen des autonomen Fahrens gefunden werden. Um diese Vielfalt der Möglichkeiten zu beherrschen, werden für die Varianten Stellvertreter gesucht. Einerseits bilden die Stellvertreter die Variationsbreite der Unterscheidungsmerkmale, die im Folgenden (Abschn. 2.4) definiert werden, ab. Andererseits stehen sie für typische Einsatzszenarien des autonomen Fahrens. Sie werden im Weiteren als Use-Cases des autonomen Fahrens bezeichnet. Um bei den Lesern und allen an dieser Diskussion Beteiligten ein gemeinsames Verständnis des autonomen Fahrens zu schaffen, werden ausgewählte Use-Cases benannt und zusätzlich durch Unterscheidungsmerkmale beschrieben. Ferner werden die vier abgeleiteten Use-Cases als Referenzbeispiele für weitere Betrachtungen innerhalb dieses Buches dienen, immer in dem Bewusstsein, dass eine Vielzahl weiterer Use-Cases existiert. Andere Use-Cases sollen damit sowohl für dieses Buch als auch für die Zukunft des autonomen Fahrens nicht ausgeschlossen werden. 
Die in diesem Kapitel getroffenen Definitionen und Annahmen können zusätzlich für die unterschiedlichen Arbeitsbereiche durch detaillierte Beschreibungen erweitert werden, da jene für die verschiedenen Arbeitsbereiche in unterschiedlicher Weise relevant sind. Das Besitzverhältnis ist beispielsweise für eine technische Betrachtung weniger relevant als für eine Betrachtung der Marktauswirkungen. Deshalb sind Definitionen und Annahmen für den Einsatz in den unterschiedlichen Arbeitsbereichen kritisch zu prüfen.

Die Herleitung und Beschreibung der Use-Cases sind in drei Abschnitte unterteilt: Zunächst werden in Abschn. 2.2 Einschränkungen und Annahmen beschrieben, die übergeordnet für alle Use-Cases gelten. Anschließend folgt in Abschn. 2.3 die Beschreibung der vier ausgewählten Use-Cases hinsichtlich ihrer Merkmalsausprägungen. Zur weiteren Vertiefung für interessierte Leser werden in Abschn. 2.4 die eingesetzten Merkmale zur Beschreibung der Use-Cases motiviert und erläutert. Zur Klärung von eventuellen Verständnisproblemen aufgrund unbekannter Begriffsdefinitionen werden die den Use-Cases zugrunde liegenden Begrifflichkeiten in Abschn. 2.5 erklärt. Dieser Aufbau wurde gewählt, um den Einstieg in die Use-Case-Beschreibung zu erleichtern; Unklarheiten in den ersten Abschnitten klären sich meist durch den Blick in Abschn. 2.5 (Grundlegende Definitionen) und Abschn. 2.4 (Ausgewählte Merkmale zur Beschreibung der Use-Cases).

\subsection{Getroffene Annahmen}

Folgende Annahmen werden für alle vorgestellten Use-Cases gleichermaßen getroffen:

Mischbetrieb: Die Use-Cases werden zum Betrachtungszeitraum in einem Mischbetrieb aus Verkehrsmitteln mit unterschiedlichen Automatisierungsgraden eingesetzt. Der Straßenverkehr besteht aus Fahrzeugen mit allen Automatisierungsgraden von ,driveronly“ über ,assistiert“ bis „vollautomatisiert“ (nach BASt [1]). Durch die stufenweise Einführung der Automatisierung ist eine menschliche Fahrzeugführung gleichzeitig zur Fahrzeugführung durch Fahrroboter wahrscheinlich.

Technisches Ausfallrisiko: Hardware-Ausfälle und Software-Fehler können auch bei autonom fahrenden Fahrzeugen auftreten. Allerdings wird davon ausgegangen, dass das nach aktuellem Stand der Technik (z. B. ISO 26262) entwickelte autonome Fahrzeug hinsichtlich der genannten Ausfälle mindestens so zuverlässig und sicher ist, wie es heutige konventionelle Fahrzeuge sind.

Detaillierungsgrad: Die Beschreibung der Use-Cases ist keine detaillierte Spezifikation. Statt einer detaillierten Beschreibung von Wetterbedingungen, Lichtverhältnissen, Fahrbahnbeschaffenheiten usw. wird vereinfachend angenommen, dass die Qualität sowie die Erfolgsquote, mit der ein Fahrroboter die Fahraufgabe ausführt, der menschlichen Qualität und Erfolgsquote ähnlich sind. Beispielsweise führt starker Regen erst dann zu einem Überführen in den sicheren Zustand und der Unterbrechung der Transportaufgabe, wenn auch der Fahrer die Fahrt unterbrechen würde. In diesem Kapitel bleibt unbeantwortet, ob diese Annahme aus Sicht der Nutzer, der Gesellschaft usw. ausreichend ist. Außerdem bleibt in diesem Kapitel offen, wie diese Qualität und Erfolgsquote quantifiziert und nachgewiesen werden könnte. 
Gesetzeskonformität: Für alle Use-Cases wird angenommen, dass die autonome Fahrt konform zum Regelwerk des Rechtsraums (Nationalstaat, bundesstaatliche Ebene in den USA) durchgeführt wird, in dem die aktuelle Fahrt stattfindet. Aus dieser Annahme folgt direkt die Frage nach dem Handeln in Dilemma-Situationen: Ist es dem Fahrroboter erlaubt bzw. besitzt er die Möglichkeit, geltende Regeln zu missachten, um einen größeren Schaden zu vermeiden? Für die Use-Cases wird angenommen, dass es einen rechtsgültigen Satz von Regeln bzw. Metaregeln gibt, die der Fahrroboter beachtet. Dafür existiert eine Freigabe von jenem Staat zur Ausführung des autonomen Fahrens, wobei in diesem Kapitel offen gelassen wird, wie diese Freigabe zu erwerben ist und wie diese Regeln lauten.

\subsection{Beschreibung der Use-Cases}

Wie bereits erwähnt, stellen Use-Cases typische Einsatzszenarien autonomen Fahrens dar; sie sind Stellvertreter einer Variationsvielfalt von möglichen Ausprägungen autonomen Fahrens. Im Folgenden wurde die Anzahl auf vier Use-Cases reduziert, wobei die Existenz weiterer Stellvertreter hierdurch nicht ausgeschlossen wird. Diese vier Use-Cases lauten:

- Autobahnautomat mit Verfügbarkeitsfahrer - Autobahnpilot

- Autonomes Valet-Parken

- Vollautomat mit Verfügbarkeitsfahrer

- Vehicle-on-Demand

Bei der automatisierten Fahrt jeder dieser vier Use-Cases handelt es sich um die Automatisierung Level 4 - vollautomatisiert nach BASt [1]. Besonders die Aufteilung der Fahraufgabe zwischen Mensch und Fahrroboter, in der sich die vier Varianten voneinander unterscheiden, hat zur Auswahl dieser Use-Cases beigetragen. Die ersten zwei Use-Cases sind als Einstiegsvarianten des autonomen Fahrens denkbar, wohingegen die zwei letzten UseCases perspektivische Varianten darstellen.

\subsubsection{Autobahnautomat mit Verfügbarkeitsfahrer - Autobahnpilot}

\subsubsection{Nutzen}

Der Fahrroboter übernimmt ausschließlich auf Autobahnen oder autobahnähnlichen Schnellstraßen die Fahraufgabe (s. Abb. 2.1). Der Fahrer wird während der autonomen Fahrt zum Passagier und bekommt die Möglichkeit, seine Hände bzw. Füße vom Lenkrad bzw. von der Pedalerie zu nehmen und einer anderen Tätigkeit nachzugehen.

\subsubsection{Beschreibung}

Ab Autobahnbeginn bzw. der Auffahrt wird dem Fahrer die Möglichkeit geboten, den Fahrroboter zu aktivieren, wobei dies sinnvollerweise in Verbindung mit der Übergabe einer Zieladresse einhergeht. Der Fahrroboter übernimmt die Navigation, Bahnführung und 


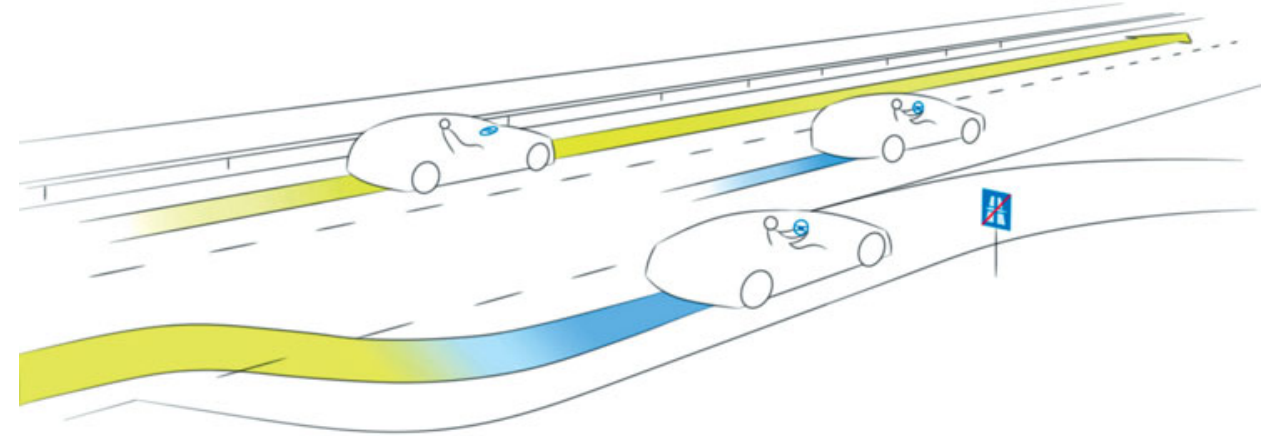

Abb. 2.1 Autobahnautomat mit Verfügbarkeitsfahrer - Autobahnpilot

Regelung so lange, bis eine Autobahnausfahrt oder ein Autobahnende erreicht bzw. bis er vom Fahrer absichtlich entbunden wird. Die Übergabe an den Fahrer wird vom Fahrroboter sicher koordiniert. Erfüllt der Fahrer nicht die Anforderungen an die sichere Übergabe - z. B. weil er schläft oder ein fehlendes Situationsbewusstsein zeigt - überführt der Fahrroboter das Fahrzeug in den risikominimalen Zustand auf dem Seitenstreifen oder kurz außerhalb der Autobahn. Während der autonomen Fahrt ist kein Situationsbewusstsein vom Insassen gefordert, es gilt die Definition nach BASt [1] für die vollautomatisierte Fahrt. Besonders die Einschränkungen der Szenerie (z. B. durch das Fehlen von Lichtsignalanlagen) sowie der anzunehmenden dynamischen Objekte (z. B. im Normalfall keine Fußgänger) führen zu einer Vereinfachung, die aus dem Use-Case ein Einstiegsszenario machen könnten, auch wenn die hohe Einsatzgeschwindigkeit das Erreichen eines risikominimalen Zustands erheblich erschwert.

\subsubsection{Merkmalsausprägungen}

Tab. 2.1 Ausprägungen des Autobahnpiloten

\begin{tabular}{|l|l|l|l|l|}
\hline \multicolumn{2}{|l|}{ Merkmal } & \multicolumn{2}{l|}{ Ausprägung } \\
\hline A & Art des Beförderten & 3. & Person/-en mit abgestimmten Zielen \\
\hline B & maximal zulässige Gesamtmasse & $1-3$. & $500 \mathrm{~kg} \mathrm{bis} 8 \mathrm{t}$ \\
\hline C & Einsatzhöchstgeschwindigkeit & 4. & bis $120 \mathrm{~km} / \mathrm{h}$ \\
\hline D & Szenerie & $8 . \mid \mathrm{a}$. & Autobahn $\quad$ ohne Freigabe erlaubt \\
\hline E & dynamische Elemente & 2. & nur Kraftfahrzeuge \\
\hline F & $\begin{array}{l}\text { Informationsfluss zwischen Fahr- } \\
\text { roboter und anderen Instanzen }\end{array}$ & $1-4$. & $\begin{array}{l}\text { optimierte Navigation, optimierte Bahnfüh- } \\
\text { rung, optimierte Regelung und Bereitstellen } \\
\text { von Umweltinformationen }\end{array}$ \\
\hline G & Verfügbarkeitskonzept & & Verfügbarkeitsfahrer \\
\hline H & $\begin{array}{l}\text { Erweiterungskonzept } \\
\text { I }\end{array}$ & Eingriffsmöglichkeiten & 2. & Fahrer \\
\hline
\end{tabular}




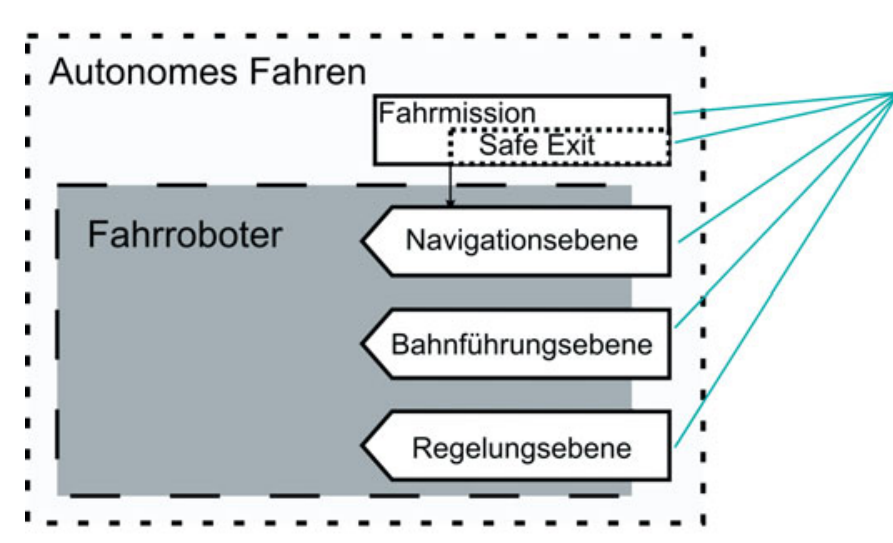

dominant

Fahrzeugnutzer

Dienstleister - Taxiuntern.

Wege- und Sonderrecht

Verkehrsleitung

Straßenverkehrsbehörde

Fahrzeughersteller

andere Verkehrsteilnehmer

rezessiv

Abb. 2.2 Eingriffsmöglichkeiten beim Autobahnpilot

Abbildung 2.2 illustriert die Eingriffsmöglichkeiten der Instanzen auf die Ebenen der Fahraufgabe für den Use-Case Autobahnpilot. Der Fahrzeugnutzer bildet hierbei die einzige Instanz, die die Möglichkeit und Befugnis besitzt, einzugreifen. Dabei sei noch einmal hervorgehoben, dass die Übergabe durch den Fahrroboter sicher gestaltet wird. Eventuelle Dienstleister, Polizei und Krankenwagen mit Wegerecht, eine Verkehrsleitung usw. besitzen keine Möglichkeit, in die Fahrzeugführung einzugreifen. Eine ausführliche Beschreibung der verwendeten Merkmale erfolgt in Abschn. 2.4.

\subsubsection{Autonomes Valet-Parken}

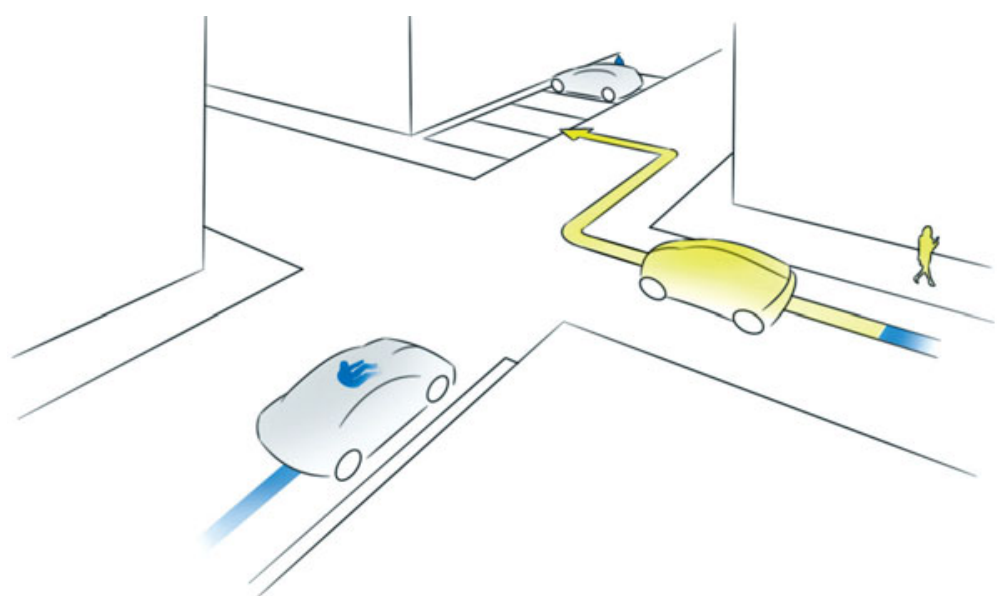

Abb. 2.3 Autonomes Valet-Parken 


\subsubsection{Nutzen}

Der Fahrroboter stellt das Fahrzeug nach Verlassen der Passagiere und dem Ausladen von Transportgut in einer nahen oder auch entfernten Parkposition ab (s. Abb. 2.3). Der Fahrroboter fährt das Fahrzeug wieder von der Parkposition an eine Wunschadresse und besitzt die Möglichkeit und Berechtigung umzuparken. Der Fahrer spart die Zeit für die Parkplatzsuche, das Abstellen sowie die Fußwege eines entfernteren Parkplatzes. Außerdem wird der Zugang zum Fahrzeug erleichtert (räumlich wie zeitlich). Zusätzlich wird der Parkraum besser genutzt und die Parkplatzsuche effizienter gestaltet.

\subsubsection{Beschreibung}

Erreicht ein Fahrer sein Fahrtziel (z. B. Arbeitsplatz, Sportverein, Wohnung), stoppt dieser das Fahrzeug, steigt aus und gibt dem Fahrroboter den Befehl, das Fahrzeug abzustellen. Somit kann der Fahrroboter das Fahrzeug nun zu einem privaten Parkplatz, einem öffentlichen Abstellplatz oder einem Parkplatz des Dienstleisters wie z. B. einer CarsharingFlotte bringen. Wichtig hierbei ist, dass dem Fahrroboter ein Parkareal zugewiesen wird, da der Parksuchverkehr durch den Fahrroboter für diesen Use-Case ausgeschlossen wird. Somit gibt es immer ein definiertes Ziel für den Fahrroboter. Ist das Fahrzeug abgestellt, ist das Umparken aufgrund der Anweisung einer autorisierten Parkplatzverwaltung möglich. Wird das Fahrzeug wieder benötigt, teilt ein autorisierter Nutzer des Fahrzeugs dem Fahrroboter eine Abholadresse mit. Der Fahrroboter fährt das Fahrzeug an diese Zieladresse und hält an, sodass der Fahrer einsteigen und die Fahraufgabe übernehmen kann.

Aufgrund der geringen Geschwindigkeit und der begrenzt befahrbaren Szenerie ist der Einsatzbereich des autonomen Valet-Parkens auf eine gewisse Umgebung um den Ort, an dem der Fahrer das Fahrzeug verlassen hat oder er seine Abholung wünscht, begrenzt. Auf der einen Seite reduzieren diese Randbedingungen die Anforderungen an die (Fahr-)Fähigkeiten des Fahrroboters erheblich, denn mit der geringen Geschwindigkeit ergibt sich eine geringere kinetische Energie sowie ein kürzerer Anhalteweg. Auf der anderen Seite könnten diese Randbedingungen dazu führen, dass das Fahrzeug als Verkehrsbehinderung und daher als lediglich begrenzt nützlich angesehen wird. Dieser Use-Case wird als Einstiegsszenario betrachtet. 


\subsubsection{Merkmalsausprägung}

Tab. 2.2 Ausprägungen des autonomen Valet-Parkens

\begin{tabular}{|c|c|c|c|c|}
\hline \multicolumn{2}{|c|}{ Merkmal } & \multicolumn{3}{|c|}{ Ausprägung } \\
\hline A & Art des Beförderten & 1. & kein Fördergut und keine Person & \\
\hline B & maximal zulässige Gesamtmasse & $1-3$ & $500 \mathrm{~kg}$ bis $8 \mathrm{t}$ & \\
\hline $\mathrm{C}$ & Einsatzhöchstgeschwindigkeit & 2. & bis $30 \mathrm{~km} / \mathrm{h}$ & \\
\hline $\mathrm{D}$ & Szenerie & $\begin{array}{l}\text { 3. } \mid \mathrm{a} . \\
\text { 4. } \mathrm{a} \text {. } \\
\text { 5. } \mid \mathrm{a} .\end{array}$ & $\begin{array}{l}\text { Parkplatz bzw. Parkhaus, } \\
\text { Erschließungsstraße, } \\
\text { angebaute Hauptverkehrsstraße }\end{array}$ & $\begin{array}{l}\text { ohne } \\
\text { Freigabe } \\
\text { erlaubt }\end{array}$ \\
\hline $\mathrm{E}$ & dynamische Elemente & 1. & ohne Ausschluss & \\
\hline $\mathrm{F}$ & $\begin{array}{l}\text { Informationsfluss zwischen Fahr- } \\
\text { roboter und anderen Instanzen }\end{array}$ & $\begin{array}{l}\text { 1. \& } \\
\text { 3. \& } \\
6 .\end{array}$ & $\begin{array}{l}\text { optimierte Navigation } \\
\text { optimierte Regelung } \\
\text { Fahrroboterüberwachung }\end{array}$ & \\
\hline G & Verfügbarkeitskonzept & 1. & keine Verfügbarkeitsergänzung & \\
\hline $\mathrm{H}$ & Erweiterungskonzept & 2. & Fahrer & \\
\hline I & Eingriffsmöglichkeiten & & siehe Abb. 2.4 & \\
\hline
\end{tabular}

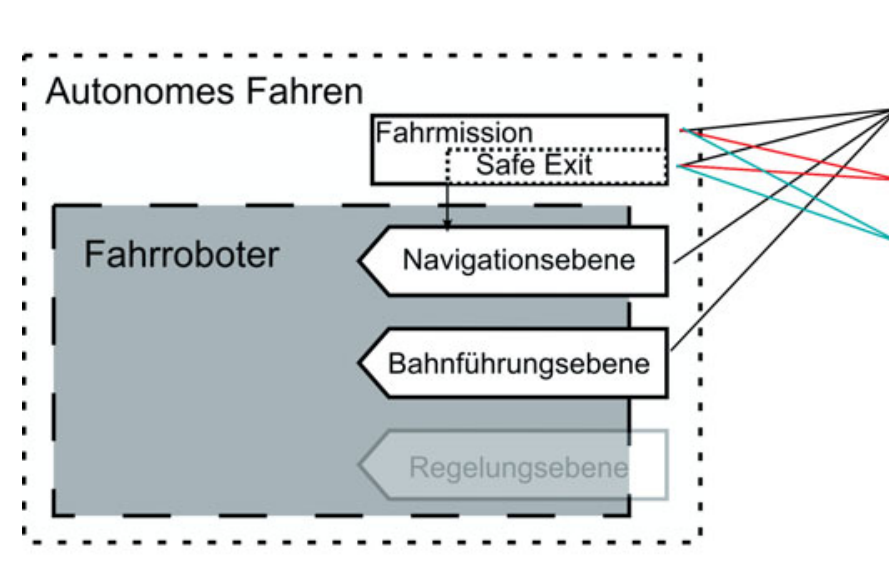

\section{dominant}

Wege- und Sonderrecht

Dienstleister - Valet-Parken

Fahrzeugnutzer

................

Straßenverkehrsbehörde

Fahrzeughersteller

andere Verkehrsteilnehmer

rezessiv

Abb. 2.4 Eingriffsmöglichkeiten beim autonomen Valet-Parken

Die Instanzen, die in die Fahraufgabe eingreifen können, sind in Abb. 2.4 auf der rechten Seite nach Rangordnung von oben (dominant) nach unten (rezessiv) aufgeführt. Der Fahrzeugnutzer besitzt die Möglichkeit, als externe Größe die Fahrmission zu ändern und dem Fahrroboter einen sogenannten Safe-Exit zu befehlen. Der Dienstleister überstimmt den Fahrzeugnutzer und kann ebenfalls auf die Fahrmission und den Safe-Exit einwirken. Beide Instanzen werden von den Instanzen mit Wege- und Sonderrecht (z. B. Polizei, Krankenwagen) überstimmt. Diese können auf Bahnführungsebene das Fahrzeug abbremsen, Navigation und Fahrmission ändern und einen Safe-Exit befehlen. 


\subsubsection{Vollautomat mit Verfügbarkeitsfahrer}

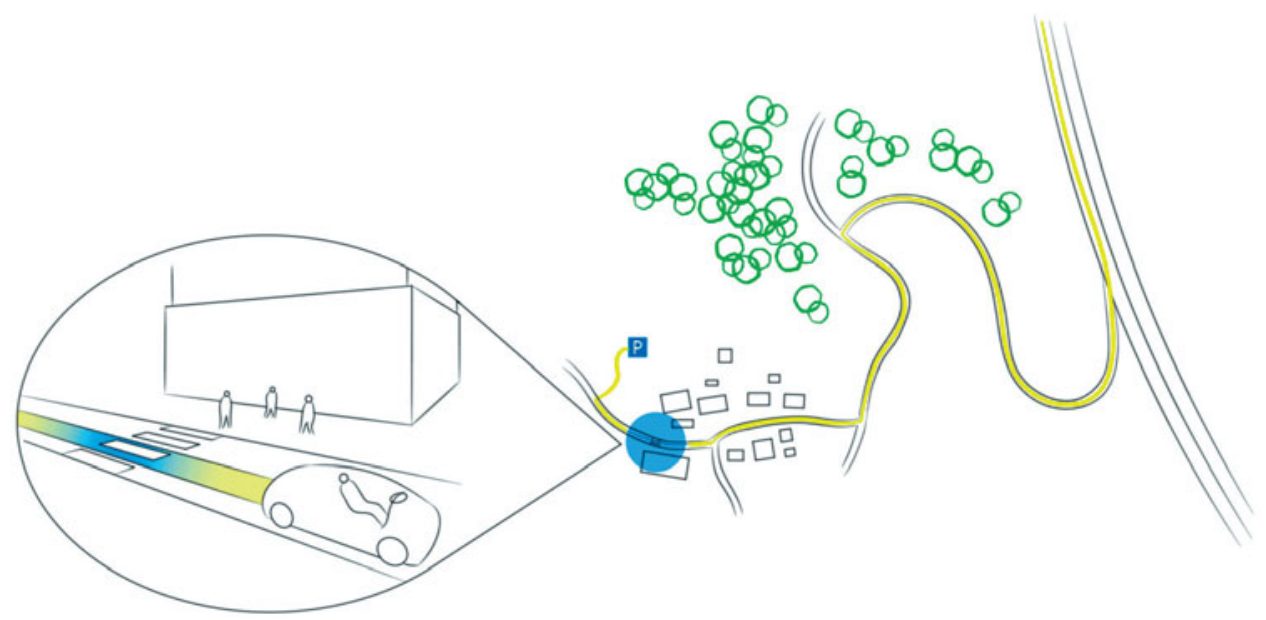

Abb. 2.5 Vollautomat mit Verfügbarkeitsfahrer

\subsubsection{Nutzen}

Der Fahrer besitzt die Möglichkeit, in den freigegebenen Bereichen die Fahraufgabe an den Fahrroboter zu übergeben. Der Fahrer wird während der autonomen Fahrt zum Passagier und hat die Möglichkeit, seine Hände bzw. Füße vom Lenkrad bzw. von der Pedalerie zu nehmen sowie einer anderen Tätigkeit nachzugehen.

\subsubsection{Beschreibung}

Die Fahraufgabe kann vom Fahrer an den Fahrroboter übergeben werden, wenn die Szenerie, in der er sich befindet, für einen autonomen Fahrbetrieb freigegeben ist. Nahezu der gesamte Verkehrsbereich im zulassenden Land ist für das Fahrzeug freigegeben, jedoch steht diese Freigabe unter dem Vorbehalt einer Eingrenzung. Wenn beispielsweise die Straßenführung geändert oder ein neues Parkhaus eröffnet wird, so könnten diese Bereiche bis zur Freigabe kurzzeitig nicht autonom befahrbar sein. Auch erscheint es in diesem Szenario sinnvoll, dass Streckenabschnitte permanent oder temporär von der Freigabe ausgenommen sind, z. B. Strecken mit einer hohen Fußgängerüberquerfrequenz. Auch hier muss die Übergabe zwischen Fahrer und Fahrroboter in sicherer Weise geschehen.

Dieser Use-Case dürfte den heutigen Vorstellungen des autonomen Fahrens am nächsten kommen, da er stark mit der heutigen Pkw-Nutzung übereinstimmt. Zwar ist die Fahraufgabe nahezu vollständig an den Fahrroboter delegiert, jedoch begleitet der bisherige Hauptnutzer und Fahrzeugführer diese Fahrt weiterhin. 


\subsubsection{Merkmalsausprägung}

Tab. 2.3 Ausprägungen des Vollautomaten mit Verfügbarkeitsfahrer

\begin{tabular}{|c|c|c|c|c|}
\hline \multicolumn{2}{|c|}{ Merkmal } & \multicolumn{3}{|c|}{ Ausprägung } \\
\hline A & Art des Beförderten & 3. & Person/-en mit abgestimmten Ziel & \\
\hline B & maximal zulässige Gesamtmasse & $1-2$. & $500 \mathrm{~kg}$ bis $2 \mathrm{t}$ & \\
\hline $\mathrm{C}$ & Einsatzhöchstgeschwindigkeit & 5. & bis $240 \mathrm{~km} / \mathrm{h}$ & \\
\hline $\mathrm{D}$ & Szenerie & $\begin{array}{l}\text { 2.|b.- } \\
\text { 8. b. }\end{array}$ & $\begin{array}{l}\text { Wirtschaftsweg, Parkplatz bzw. } \\
\text { Parkhaus, Erschließungsstraße, } \\
\text { angebaute Hauptverkehrsstraße, } \\
\text { anbaufreie Hauptverkehrsstraße, } \\
\text { Landstraße, Autobahn }\end{array}$ & $\begin{array}{l}\text { mit } \\
\text { Freigabe } \\
\text { erlaubt }\end{array}$ \\
\hline $\mathrm{E}$ & dynamische Elemente & 1. & ohne Ausschluss & \\
\hline $\mathrm{F}$ & $\begin{array}{l}\text { Informationsfluss zwischen Fahr- } \\
\text { roboter und anderen Instanzen }\end{array}$ & $1-6$. & $\begin{array}{l}\text { optimierte Navigation, optimierte } \\
\text { führung, optimierte Regelung, Be } \\
\text { von Umweltinformationen, Aktua } \\
\text { der Fahrroboterfähigkeit, Fahrrob } \\
\text { wachung }\end{array}$ & $\begin{array}{l}\text { Bahn- } \\
\text { eitstellen } \\
\text { isierung } \\
\text { terüber- }\end{array}$ \\
\hline G & Verfügbarkeitskonzept & 2. & Verfügbarkeitsfahrer & \\
\hline $\mathrm{H}$ & Erweiterungskonzept & 2. & Fahrer & \\
\hline I & Eingriffsmöglichkeiten & & siehe Abb. 2.6 & \\
\hline
\end{tabular}

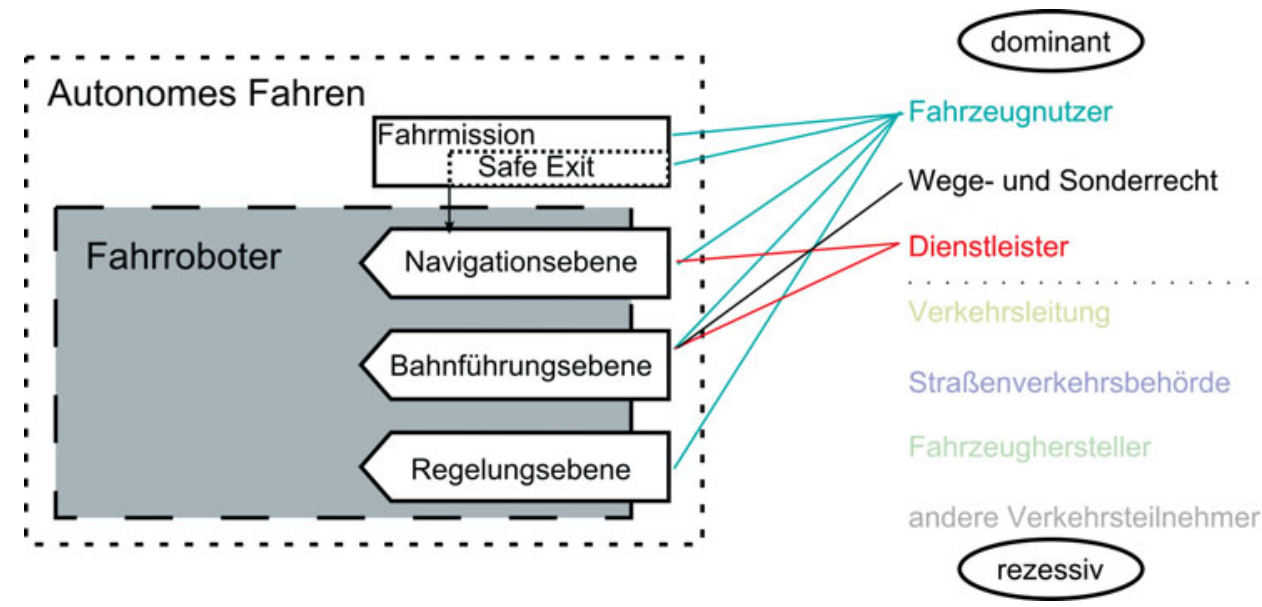

Abb. 2.6 Eingriffsmöglichkeiten beim Vollautomaten mit Verfügbarkeitsfahrer

In Abb. 2.6 wird dargestellt, welche Instanz (rechts) auf welche Ebene der Fahraufgabe (links) eingreifen kann. Der Fahrzeugnutzer kann, wenn gewünscht, nach sicherer Übergabe durch den Fahrroboter das Fahrzeug wie ein klassisches Driver-Only-Automobil 
fahren. Des Weiteren existieren Schnittstellen für die Bahnführungsebene, Navigationsebene und die Fahrmission, sodass der Fahrer auch auf diesen Ebenen Eingriffsmöglichkeiten besitzt. Der Fahrzeugnutzer dominiert die Instanzen mit Wege- und Sonderrecht. Polizei und Krankenwagen, die die Berechtigung besitzen, auf Bahnführungsebene einzugreifen, können somit vom Fahrzeugnutzer überstimmt werden. Entsprechendes gilt für einen Dienstleister: Der Dienstleister könnte auf Navigation und Bahnführung eingreifen, solange er nicht überstimmt wird. Für welche Dienste der Dienstleister diesen Zugriff benötigt, bleibt in diesem Kapitel unbeantwortet. Jedoch sind Konzepte denkbar, bei denen ein Dienstleister für Werbezwecke die Navigation übernimmt und dafür einen Teil der Kraftstoffkosten bzw. der Reisekosten trägt.

\subsubsection{Vehicle-on-Demand}

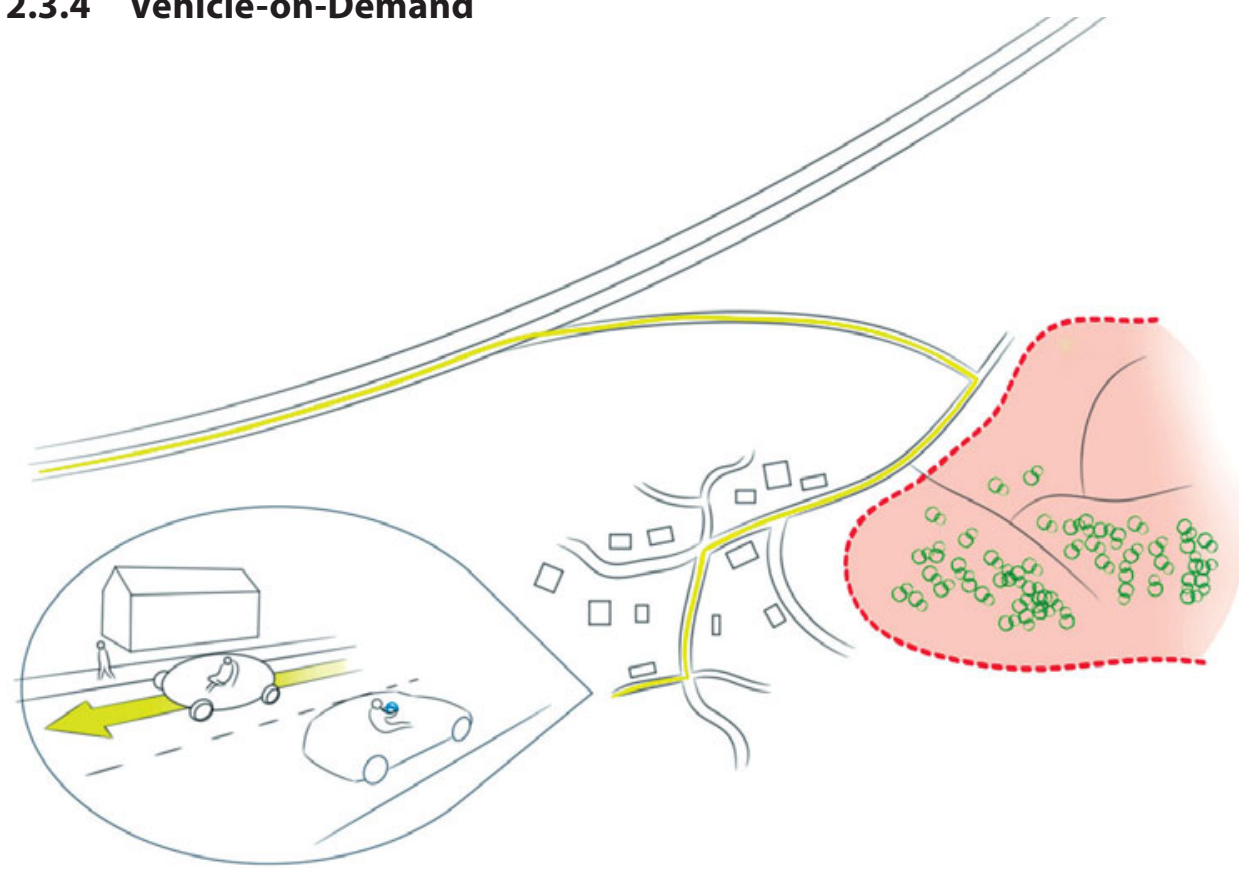

Abb. 2.7 Vehicle-on-Demand

\subsubsection{Nutzen}

Der Fahrroboter bewegt das Fahrzeug in allen Szenerien mit Insassen, mit Fördergut oder aber auch komplett ohne Ladeinhalt/Insassen autonom (s. Abb. 2.7). Passagiere nutzen die Fahrzeit komplett frei und für andere Dinge als die Bewältigung der Fahraufgabe. Der Innenraum ist hierbei völlig frei gestaltet. Transportgut kann ohne Pause nahezu 24 Stunden am Tag mithilfe des Fahrroboters transportiert werden, sofern dies durch die Fahrenergieversorgung nicht eingeschränkt wird. 


\subsubsection{Beschreibung}

Der Fahrroboter bekommt von Insassen bzw. externen Größen (Nutzer, Dienstleister usw.) Fahrtziele mitgeteilt, zu denen er das Fahrzeug autonom befördert. Für den Menschen existiert keine Möglichkeit, die Fahraufgabe zu übernehmen. Er besitzt lediglich die Möglichkeit, das Fahrtziel zu bestimmen oder den Safe-Exit zu aktivieren. Verschiedene Geschäftsmodelle sind mit diesem Fahrroboter denkbar: Eine Mischung aus Taxi-Service und Carsharing, autonome Transportfahrzeuge oder sogar Nutzungsmodelle, die über die alleinige Transportaufgabe hinausgehen. Ein Beispiel hierfür ist ein Fahrzeug für soziale Netzwerke, das direkt Informationen aus dem Netzwerk nutzt, um Routen zu planen und Personen zusammenzubringen, oder das weitere Dienstleistungen anbietet, an die heute noch nicht gedacht wird.

\subsubsection{Merkmalsausprägung}

Tab. 2.4 Ausprägungen des Vehicle-on-Demand

\begin{tabular}{|c|c|c|c|c|}
\hline \multicolumn{2}{|c|}{ Merkmal } & \multicolumn{3}{|c|}{ Ausprägung } \\
\hline A & Art des Beförderten & $1-4$ & \multicolumn{2}{|c|}{$\begin{array}{l}\text { kein Fördergut und keine Person, } \\
\text { für Transport aufgegebenes Fördergut, } \\
\text { Person/-en mit abgestimmten Zielen, } \\
\text { Personen mit nicht abgestimmten Zielen }\end{array}$} \\
\hline B & maximal zulässige Gesamtmasse & $1-3$ & \multicolumn{2}{|l|}{ von $500 \mathrm{~kg}$ bis $8 \mathrm{t}$} \\
\hline $\mathrm{C}$ & Einsatzhöchstgeschwindigkeit & 4. & \multicolumn{2}{|l|}{ bis $120 \mathrm{~km} / \mathrm{h}$} \\
\hline $\mathrm{D}$ & Szenerie & $\begin{array}{l}\text { 2.|a.- } \\
\text { 8.|a. }\end{array}$ & $\begin{array}{l}\text { Wirtschaftsweg, Parkplatz bzw. } \\
\text { Parkhaus, Erschließungsstraße, } \\
\text { angebaute Hauptverkehrsstraße, } \\
\text { anbaufreie Hauptverkehrsstraße, } \\
\text { Landstraße, Autobahn }\end{array}$ & $\begin{array}{l}\text { ohne } \\
\text { Freigabe } \\
\text { erlaubt }\end{array}$ \\
\hline $\mathrm{E}$ & dynamische Elemente & 1. & \multicolumn{2}{|l|}{ ohne Ausschluss } \\
\hline $\mathrm{F}$ & $\begin{array}{l}\text { Informationsfluss zwischen Fahr- } \\
\text { roboter und anderen Instanzen }\end{array}$ & $1-8$ & \multicolumn{2}{|c|}{$\begin{array}{l}\text { optimierte Navigation, optimierte Bahn- } \\
\text { führung, optimierte Regelung, Bereitstellen } \\
\text { von Umweltinformationen, Fahrroboterüber- } \\
\text { wachung, } \\
\text { Insassenüberwachung, Insassen Notruf }\end{array}$} \\
\hline G & Verfügbarkeitskonzept & 3. & \multicolumn{2}{|l|}{ teleoperiertes Fahren } \\
\hline $\mathrm{H}$ & Erweiterungskonzept & 1. & \multicolumn{2}{|l|}{ keine Erweiterung } \\
\hline I & Eingriffsmöglichkeiten & & \multicolumn{2}{|l|}{ siehe Abb. 2.8} \\
\hline
\end{tabular}

Die Eingriffsmöglichkeiten auf den Use-Case Vehicle-on-Demand sind besonders aufgrund der weitreichenden (Fahr-)Fähigkeiten des Fahrroboters umfangreich: Die Regelungsebene wird dauerhaft durch den Fahrroboter ausgeführt. Die zwei Instanzen Verkehrsleitung sowie Wege- und Sonderrecht (Instanz mit größter Dominanz) können auf Navigations- und Bahnführungsebene eingreifen. Fahrzeugnutzer und Dienstleister besitzen 


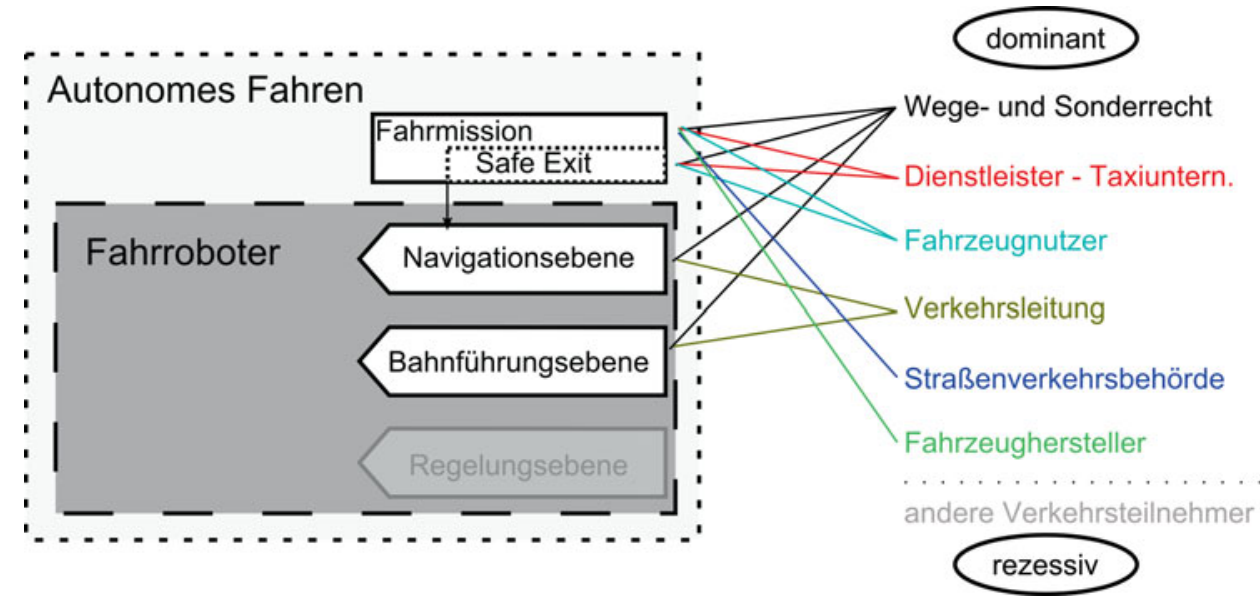

Abb. 2.8 Eingriffsmöglichkeiten beim Vehicle-on-Demand

Zugriff auf den Safe-Exit, sodass ein Insasse das Fahrzeug sicher und schnellstmöglich verlassen kann. Besonders hervorzuheben ist hier, dass der Fahrzeugnutzer vom Dienstleister und der Instanz mit Wege- und Sonderrecht überstimmt werden kann. Überstimmt eine der Instanzen den Nutzer, kann dieser auch den Safe-Exit nicht mehr bedienen und ist gezwungen, weiter im Fahrzeug zu bleiben. Diese Konstellation entspricht aktuellen Taxikonzepten. Der Taxifahrer kann dem Wunsch eines Fahrgastes entsprechend und so schnell wie möglich anhalten. Prinzipiell hat er jedoch auch die Möglichkeit, diesen Wunsch zu missachten und das Fahrzeug nach eigenem Wunsch zu führen.

\subsection{Ausgewählte Merkmale zur Beschreibung der Use-Cases}

In diesem Abschnitt werden Merkmale und deren mögliche Ausprägungen vorgestellt, mit denen die ausgewählten Use-Cases klassifiziert werden können. Neben den im Folgenden beschriebenen technisch orientierten Merkmalen können noch viele weitere Unterscheidungsmerkmale festgelegt werden, z. B. hinsichtlich Geschäftsmodell oder Marktplatzierung. Dieses soll auch aufgrund des bisher geringen Kenntnisstands über die konkreten Produkte zunächst hintangestellt werden.

Die Merkmale, in der alphabetischen Ordnung A bis I, wurden aus dem Drei-EbenenModell der Fahraufgabe nach Donges [3] abgeleitet und für die Beschreibung ausgewählt. Hierbei wird die Fahraufgabe in die Ebenen Navigation, Bahnführung und Stabilisierung aufgeteilt. 


\subsubsection{Merkmal A: Art des Beförderten}

\subsubsection{Motivation}

Für die individuelle Mobilität mit einem Fahrzeug ist heutzutage immer ein Mensch notwendig, der sich in dem Fahrzeug befindet und es dauernd bzw. unter allen Umständen beherrschen muss [6]. Diese Bedingung könnte sich durch die Automatisierung der Fahraufgabe ändern. Die Art des Beförderten stellt außerdem grundlegende Anforderungen an das Fahrzeugkonzept und an das Sicherheitskonzept eines Fahrzeugs.

\subsubsection{Merkmalsausprägungen}

1. kein Fördergut und keine Person (dadurch keine spezifischen Insassen- oder Transportschutzinteressen)

2. für Transport aufgegebenes Fördergut

3. Person/-en mit abgestimmten Zielen (Individualverkehr)

4. Personen mit nicht abgestimmten Zielen (öffentlicher Verkehr)

Ein Use-Case kann durch mehrere Ausprägungen dieses Merkmals abgedeckt werden. Die Aufteilung in Ausprägung 3 und 4 wird getroffen, um Individualverkehr und öffentlichen Verkehr zu unterscheiden. Ein Fahrzeug des Individualverkehrs befördert Personen mit abgestimmten Zielen. Im Gegensatz dazu befördert ein Fahrzeug des öffentlichen Verkehrs zusätzlich Personen, die nicht zuvor ihre Ziele abgestimmt haben. Mit dem öffentlichen Verkehr erreichen die unterschiedlichen Personen dennoch ihre Ziele, da ein Fahrplan mit Zielen und Zwischenzielen feststeht.

\subsubsection{Merkmal B: Maximal zulässige Gesamtmasse}

\subsubsection{Motivation}

Die maximal zulässige Gesamtmasse geht über die kinetische Energie in die Sicherheitsbetrachtung ein. Neben der Sicherheitsbetrachtung erweitert die Betrachtung der Gesamtmasse die Diskussion über den Individualverkehr bis hin zum öffentlichen Verkehr, den Gütertransport und die straßenbauliche Infrastruktur. Zusätzlich adressiert dieses Merkmal auf einer hohen Abstraktionsebene die Frage nach den Fahrzeugtypen, die möglicherweise durch die autonome Fahrfunktion und die sich ändernden Anforderungen nicht mit den aktuellen Fahrzeugtypen übereinstimmen werden. Statt auf die Grenzen der oft länderspezifischen Fahrzeugklassen einzugehen, werden vier Massenangaben gewählt, die von ultraleichten Fahrzeugen bis zu Schwerlastkraftwagen repräsentierenden Werten reichen und jeweils einen Faktor vier voneinander entfernt sind. 


\subsubsection{Merkmalsausprägungen}

1. ultraleichte Pkw um $500 \mathrm{~kg}$

2. Pkw um $2 t$

3. leichte Lkw und Transporter um $8 t$

4. schwere Lkw um 32 t

Eine diskrete Aufteilung wird eingesetzt, um die fiktiven Use-Cases zu beschreiben und ihre Masse ungefähr einzuordnen. Bei existierenden Use-Cases und definierten Einsatzbereichen ist die exakte Angabe einer Masse möglich.

\subsubsection{Merkmal C: Einsatzhöchstgeschwindigkeit}

\subsubsection{Motivation}

Das Merkmal der Einsatzhöchstgeschwindigkeit (genau genommen das Quadrat der Geschwindigkeit) bestimmt zusammen mit der Masse die maximale kinetische Energie und ist daher ebenfalls zu unterscheiden. Ferner berechnet sich der Bremsweg in den Stand über das Geschwindigkeitsquadrat. Entsprechend steigen die Anforderungen an das vollautomatisierte System für das vorausgesetzte Herbeiführen eines risikominimalen Zustandes im Fehlerfall oder an Funktionsgrenzen mit dem Geschwindigkeitsquadrat.

Neben der Sicherheitsbetrachtung sind die Reisezeit und folglich die bezogen auf den zeitlichen Aufwand noch vertretbare Reichweite als Ergebnis der Einsatzgeschwindigkeit eine die individuelle Mobilität beeinflussende Größe. Zusätzlich beschränkt die Einsatzhöchstgeschwindigkeit gegebenenfalls das Einsatzgebiet des Fahrzeugs, falls bauartbedingte Mindestgeschwindigkeiten auf bestimmten Straßentypen vorgeschrieben sind.

\subsubsection{Merkmalsausprägungen}

1. bis $5 \mathrm{~km} / \mathrm{h}$

2. bis $30 \mathrm{~km} / \mathrm{h}$

3. bis $60 \mathrm{~km} / \mathrm{h}$

4. bis $120 \mathrm{~km} / \mathrm{h}$

5. bis $240 \mathrm{~km} / \mathrm{h}$

Die Einsatzgeschwindigkeit wird in fünf Stufen unterteilt, wobei die erste Stufe Schrittgeschwindigkeit charakterisiert und die nachfolgenden sich jeweils um den Faktor zwei voneinander unterscheiden (=Faktor vier in Bezug auf kinetische Energie und Bremsweg). Eine diskrete Aufteilung wird eingesetzt, um die fiktiven Use-Cases zu beschreiben und ihre Einsatzhöchstgeschwindigkeit einzuordnen. Für konkrete Use-Cases ist ein Wert für die Einsatzhöchstgeschwindigkeit unabhängig von den fünf Stufen anzugeben. 


\subsubsection{Merkmal D: Szenerie}

\subsubsection{Motivation}

Welche räumlichen Gebiete, die dem Fahrzeugführer durch das „Driver-only“-Automobil zugänglich sind, werden auch mit dem beschriebenen Use-Case des autonomen Fahrens befahrbar? Das Merkmal Szenerie beschreibt den räumlichen Einsatzbereich, in dem das Fahrzeug autonom fährt: Existieren beispielsweise genormte Strukturen, wie viele Fahrstreifen sind vorhanden und existieren sonstige Kennzeichnungen?

Bereits die statische Szenerie kann vielseitig sein und eine Herausforderung für den Fahrroboter darstellen. Ein Beispiel dafür sind die oft erwähnten, von Schnee bedeckten Fahrstreifen oder die von Büschen oder Bäumen verdeckten Verkehrszeichen. Diese bei Fahrtbeginn eventuell unbekannten und nicht beeinflussbaren Szenerie-Setups werden nicht bei diesem Merkmal beachtet. Inwiefern der Fahrroboter diese Szenerie-Setups bewältigen kann, ist durch die Annahme beschrieben, dass die Qualität und Erfolgsquote des Fahrroboters bei der Bewältigung der Fahraufgabe denen des menschlichen Fahrers entspricht.

Dieses Merkmal beschreibt folglich auf hoher Abstraktionsebene (Lage, Umfeld und Funktion der Straße) die vorhersehbaren und Regeln folgenden Szenerien.

\subsubsection{Merkmalsausprägungen}

\section{Dimension: Art der Szenerie}

1. Gelände

2. Wirtschaftsweg

3. Parkplatz bzw. Parkhaus

4. Erschließungsstraße

5. angebaute Hauptverkehrsstraße

6. anbaufreie Hauptverkehrsstraße

7. Landstraße

8. Autobahn

9. Sonderareale

Das Merkmal Szenerie besitzt in seiner ersten Dimension neun Ausprägungen, die aus der Erweiterung der Richtlinie für integrierte Netzgestaltung [7] hervorgehen. Die Beschreibung der einzelnen Ausprägungen findet sich in Tab. 2.5.

\section{Dimension: Freigabe}

Das Merkmal Szenerie besitzt neben der Ausprägung, innerhalb welcher Szenerie der Use-Case betrieben werden kann, eine zweite Dimension. Diese Dimension bedingt, ob die Szenerie explizit freigegeben werden muss oder nicht.

a. Ohne Freigabe erlaubt: Alle Szenerien dieser Art sind für die autonome Fahrt geeignet, sodass sich der Fahrroboter in diesen Use-Cases frei bewegen kann.

b. Nur mit Freigabe erlaubt: Nur ausgewählte und freigegebene Szenerien dieser Art erlauben dem Fahrroboter die autonome Fahrt in diesem Bereich. 
Wer diese Freigabe ausführt, ob es ein privater oder staatlicher Dienst ist, wird zunächst offen gelassen. Ebenso wird zunächst die Art der Freigabe nicht weiter spezifiziert, beispielsweise könnte die Infrastruktur speziell gewartet oder eine Karte mit weiteren Informationen angereichert und bereitgestellt werden. Auch kann die Freigabe eine temporäre Komponente einbeziehen und statische oder dynamische Ausschlusszeiten für bestimmte Szeneriebereiche enthalten.

Tab. 2.5 Ausprägungen der Szenerie von Merkmal D

\begin{tabular}{|c|c|}
\hline Ausprägung & Beschreibung \\
\hline $\begin{array}{l}\text { Gelände } \\
\text { (offroad) }\end{array}$ & $\begin{array}{l}\text { - keine genormten oder bekannten Strukturen, wie Fahrstreifen oder sonstige } \\
\text { Kennzeichnungen } \\
\text { - keine erkennbare Verkehrsregelung } \\
\text { - nicht für das Befahren befestigt }\end{array}$ \\
\hline $\begin{array}{l}\text { Wirtschafts- } \\
\text { weg }\end{array}$ & $\begin{array}{l}\text { - Feldwege, Waldwege und Ähnliches } \\
\text { - oft nur minimal befestigte Fahrbahn } \\
\text { - öffentlich zugänglich } \\
\text { - festgelegte Regeln für Straßenverkehr gelten }\end{array}$ \\
\hline $\begin{array}{l}\text { Parkplatz bzw. } \\
\text { Parkhaus }\end{array}$ & $\begin{array}{l}\text { - explizit für das Parken von Fahrzeugen ausgelegt und beschildert } \\
\text { - nicht immer Fahrstreifenmarkierungen, sondern stattdessen genormte } \\
\text { Markierung der Verkehrsfläche für das geordnete Abstellen der Fahrzeuge } \\
\text { - im urbanen Raum: Parkhäuser mit mehreren Ebenen und teils schmale } \\
\text { Auffahrtsrampen und wenig Rangierfreiraum } \\
\text { - festgelegte Regeln für Straßenverkehr gelten }\end{array}$ \\
\hline $\begin{array}{l}\text { Erschließungs- } \\
\text { straße }\end{array}$ & $\begin{array}{l}\text { - angebaute Straßen innerhalb bebauter Gebiete, die im Wesentlichen } \\
\text { der unmittelbaren Erschließung der angrenzenden bebauten Grundstücke } \\
\text { oder dem Aufenthalt dienen } \\
\text { - Anbindung (flächenhafte Erschließung) der durch Wohnen, Arbeiten } \\
\text { und Versorgung geprägten Ortsteile } \\
\text { - grundsätzlich einbahnig } \\
\text { - untereinander mit plangleichen Knotenpunkten ohne Lichtsignalanlagen } \\
\text { verknüpft } \\
\text { - Verknüpfung mit angebauten Hauptverkehrsstraßen durch plangleiche } \\
\text { Knotenpunkte mit oder ohne Lichtsignalanlage oder Kreisverkehre } \\
\text { - in besonderen Fällen für öffentlichen Personenverkehr } \\
\text { - Aufnahme des wesentlichen Teils des innerörtlichen Radverkehrs } \\
\text { - festgelegte Regeln für Straßenverkehr gelten }\end{array}$ \\
\hline $\begin{array}{l}\text { angebaute } \\
\text { Hauptver- } \\
\text { kehrsstraße }\end{array}$ & $\begin{array}{l}\text { - angebaute Straßen innerhalb bebauter Gebiete, die im Wesentlichen der } \\
\text { Verbindung dienen bzw. den Verkehr aus Erschließungsstraßen sammeln } \\
\text { - in der Regel Aufnahme von Linien des öffentlichen Personenverkehrs } \\
\text { - z. T. Bestandteile zwischengemeindlicher Verbindungen } \\
\text { (Ortsdurchfahrten) } \\
\text { - einbahnig oder zweibahnig } \\
\text { - Verknüpfung mit Straßen der gleichen Kategoriengruppe i.A. durch } \\
\text { plangleiche Knotenpunkte mit Lichtsignalanlage oder Kreisverkehren } \\
\text { - z. T. durch Flächen des ruhenden Verkehrs geprägt } \\
\text { - festgelegte Regeln für Straßenverkehr gelten }\end{array}$ \\
\hline
\end{tabular}


Tab. 2.5 (Fortsetzung)

\begin{tabular}{|c|c|}
\hline Ausprägung & Beschreibung \\
\hline $\begin{array}{l}\text { anbaufreie } \\
\text { Hauptver- } \\
\text { kehrsstraße }\end{array}$ & $\begin{array}{l}\text { - anbaufreie Straßen im Vorfeld oder innerhalb bebauter Gebiete } \\
\text { - Verbindungsfunktionen } \\
\text { - Straßenseitenräume mit lockeren Bebauung und Einrichtungen der tertiären } \\
\text { Nutzung }{ }^{1} \text {, daher geringe Erschließungsfunktion } \\
\text { - einbahnig oder zweibahnig } \\
\text { - Verknüpfung mit dem übrigen Straßennetz überwiegend durch plangleiche } \\
\text { Knotenpunkte mit Lichtsignalanlage oder Kreisverkehren } \\
\text { - festgelegte Regeln für Straßenverkehr gelten }\end{array}$ \\
\hline Landstraße & $\begin{array}{l}\text { - anbaufreie Straßen außerhalb bebauter Gebiete } \\
\text { - einbahnig mit kurzen zweibahnigen Abschnitten } \\
\text { - Verknüpfung mit Straßen der gleichen Kategoriengruppe i.A. durch plan- } \\
\text { gleiche oder planfreie Knotenpunkte } \\
\text { - im Wesentlichen Verbindungsfunktion } \\
\text { - bei einzelnen unmittelbar an die Straße angrenzenden Gebäuden in geringem } \\
\text { Maße Erschließungsfunktion } \\
\text { - festgelegte Regeln für Straßenverkehr gelten }\end{array}$ \\
\hline Autobahn & $\begin{array}{l}\text { - anbaufreie Straßen außerhalb, im Vorfeld und innerhalb bebauter Gebiete } \\
\text { - planfreie und teilplanfreie Knotenpunkte } \\
\text { - zweibahnig } \\
\text { - ausschließlich für schnellen Kraftfahrzeugverkehr } \\
\text { - Zufahrt nur über spezielle Anschlussstellen } \\
\text { - festgelegte Regeln für Straßenverkehr gelten }\end{array}$ \\
\hline Sonderareale & $\begin{array}{l}\text { - nicht öffentlich zugänglich } \\
\text { - Geometrie ist nicht bekannt } \\
\text { - beispielsweise um weiträumiges privates Gelände oder Industrieanlagen } \\
\text { - evtl. zusätzliche Infrastruktur für autonomes Fahren (z. B. Containerhäfen } \\
\text { mit autonomen Systemen für die Be- und Entladung sowie die Kommissio- } \\
\text { nierung) }\end{array}$ \\
\hline
\end{tabular}

${ }^{1}$ d.h. Nutzung durch Handel und Gewerbe

\subsubsection{Merkmal E: Dynamische Elemente}

\subsubsection{Motivation}

Die Komplexität einer Szene hängt neben der Szenerie maßgeblich von den dynamischen Elementen ab. Die dynamischen Elemente, die sich zusätzlich zu dem autonom fahrenden Fahrzeug in der Szene befinden, erweitern die Anforderungen an die (Fahr-)Fähigkeiten des Fahrroboters. Somit beschreibt dieses Merkmal, inwieweit der Use-Case im aktuellen Straßenverkehr eingesetzt werden kann oder ob Einschränkungen bzw. der Ausschluss von dynamischen Elementen vorgesehen ist. 


\subsubsection{Merkmalsausprägungen}

1. ohne Ausschluss

2. nur Kraftfahrzeuge

3. nur autonom fahrende Fahrzeuge

4. keine anderen dynamischen Elemente

Eine Beschreibung der einzelnen Ausprägungen findet sich in Tab. 2.6. Der Ausschluss anderer dynamischer Elemente bei den Ausprägungen 2 bis 4 ist nicht ohne Ausnahme gegeben, wie das folgende Beispiel illustriert: Die Szene auf einer heutigen Autobahn wird beispielsweise durch Ausprägung 2 Nur Kraftfahrzeuge beschrieben. Der Fall, dass eine Person oder ein Radfahrer die Autobahn betritt, ist trotzdem möglich, wird aber aufgrund seiner Eintrittswahrscheinlichkeit vernachlässigt. Gemäß der in Abschn. 2.2 (Getroffene Annahmen) genannten gemeinsamen Eigenschaften (Mischbetrieb) werden nur die Ausprägungen 1 und 2 für die Use-Case-Bildung verwendet.

Tab. 2.6 Ausprägungen des Merkmals E

\begin{tabular}{|c|c|}
\hline Ausprägung & Beschreibung \\
\hline ohne Ausschluss & $\begin{array}{l}\text { - komplexeste Szene } \\
\text { - beinhaltet z. B. Tiere, Fußgänger, Radfahrer, Fahrzeuge, Polizeibeamte }\end{array}$ \\
\hline nur Kraftfahrzeuge & $\begin{array}{l}\text { - Aufeinandertreffen von autonomen Fahrzeugen und menschgeführten } \\
\text { Kraftfahrzeugen } \\
\text { - Tiere, Fußgänger usw. nur als extrem seltene Ausnahme behandelt }\end{array}$ \\
\hline $\begin{array}{l}\text { nur autonom fahrende } \\
\text { Fahrzeuge }\end{array}$ & - exklusive Szenerie für sich autonom bewegende Fahrzeuge \\
\hline $\begin{array}{l}\text { keine anderen dyna- } \\
\text { mischen Elemente }\end{array}$ & - exklusiv für ein (autonom fahrendes) Fahrzeug \\
\hline
\end{tabular}

\subsubsection{Merkmal F: Informationsfluss zwischen Fahrroboter und anderen Instanzen}

\subsubsection{Motivation}

Der Fahrroboter erfüllt die Aufgaben der Perzeption, Kognition, Verhaltensentscheidung und Verhaltensausführung: Dafür werden Informationen über den eigenen Zustand des vom Fahrroboter gesteuerten Fahrzeugs wie beispielsweise Position und Geschwindigkeit, aber auch Informationen über die Umwelt und die Insassen benötigt. Diese Informationen werden entweder durch Sensoren, durch Auslesen eines Speichers oder durch Kommunikation gewonnen. Wie und welche Informationen vom Fahrroboter mit welcher Instanz ausgetauscht werden, ist durch den Zweck des Informationsflusses definiert. Um den Informationsfluss für einen Use-Case zu beschreiben, werden den Use-Cases deshalb die Zwecke des Informationsaustausches zugeordnet. 
Die Verfügbarkeit der Information muss der Übertragung sowie den Kommunikationspartnern und dem Einsatzzweck entsprechen. Wie bereits beschrieben, wird zusätzlich davon ausgegangen, dass die Technologie nur sukzessiv Einzug in den Markt hält; somit beherrschen nicht alle dynamischen Elemente in der Umgebung den Informationsaustausch. Der hier betrachtete Informationsfluss des Fahrroboters ist eine Untermenge des gesamten Informationsflusses des Fahrzeugs. Für die Betrachtung werden an dieser Stelle Zwecke, die zu Infotainment und Komfortsteigerung zu rechnen sind, vernachlässigt. Aktuelle Nachrichten, der Zugang zu einem sozialen Netzwerk oder Musikstreamingfunktionen können als Dienstleistungen den Zusatznutzen der autonomen Fahrt steigern, jedoch ist der Informationsfluss durch diese Dienste nicht primär für das autonome Fahren relevant. Deshalb werden nur Zwecke mit Einfluss auf die Verkehrssicherheit, Verkehrseffizienz sowie Zwecke, die eventuell für die autonome Fahrt vorausgesetzt werden, als Unterscheidungsmerkmal der Use-Cases beschrieben.

\subsubsection{Merkmalsausprägungen}

\section{Optimierung der Navigation}

2. Optimierung der Bahnführung

3. Optimierung der Regelung

4. Bereitstellen von Umweltinformationen

5. Aktualisierung der Fahrroboterfähigkeit

6. Fahrroboterüberwachung

7. Insassenüberwachung

\section{Insassennotruf}

Die zu den Ausprägungen gehörigen Beschreibungen sind in Tab. 2.7 gegeben.

Die ersten drei Ausprägungen besitzen zusätzlich das Potenzial, zu Interaktionen zu führen, die einer Verhandlung über die zeitliche und räumliche Nutzung von Verkehrsinfrastruktur entsprechen. Zunächst wird aber darauf verzichtet, diese Interaktionsmöglichkeit weiter zu betrachten.

Tab. 2.7 Ausprägungen des Merkmals F

\begin{tabular}{l|l|l|l|l}
\hline Ausprägung & Beschreibung \\
\hline $\begin{array}{l}\text { Optimierung } \\
\text { der Navigation }\end{array}$ & $\begin{array}{l}\text { Austausch von für die Navigation relevanten Informationen wie Position, } \\
\text { Fahrtziel, Witterung, Fließgeschwindigkeiten usw. mit überregionaler } \\
\text { Verkehrszentrale (mehrere } 100 \text { km Einzugsgebiet) } \\
\text { - Ziele der Optimierung: geringer Energieverbrauch, } \mathrm{CO}_{2} \text {-Ausstoß, } \\
\text { möglichst kurze Fahrtzeit/Fahrstrecke etc. }\end{array}$ \\
\hline $\begin{array}{l}\text { Optimierung } \\
\text { der Bahnführung }\end{array}$ & $\begin{array}{l}\text { Austausch von ausführlichen Informationen über Zustand }(x, v, a, \ldots) \\
\text { und Intention des durch den Fahrroboter bewegten Fahrzeugs sowie der } \\
\text { Fahrzeuge in direkter Umgebung } \\
\text { - Teilen von Informationen wie Witterung, Fahrbahnbeschaffenheit, } \\
\text { Engstellen, Schaltzeiten von Lichtsignalanlagen (Ampeln) usw. mit einer } \\
\text { lokalen Verkehrszentrale (wenige km Einzugsgebiet) }\end{array}$
\end{tabular}


Tab. 2.7 (Fortsetzung)

\begin{tabular}{|c|c|}
\hline Ausprägung & Beschreibung \\
\hline $\begin{array}{l}\text { Optimierung } \\
\text { der Bahnführung }\end{array}$ & $\begin{array}{l}\text { - synchronisierte Fahrt (eine unter den benachbarten Fahrzeugen ab- } \\
\text { gestimmte Fahrt) im Quer- sowie Längsverkehr (z. B. Kolonnenfahrt, } \\
\text { verkehrsregelfreie Kreuzungen, sich anpassende Fahrstreifen etc.) als } \\
\text { beispielhafte Zielsetzung }\end{array}$ \\
\hline $\begin{array}{l}\text { Optimierung } \\
\text { der Regelung }\end{array}$ & $\begin{array}{l}\text { - Austausch von ausgewählten Fahrzeugzuständen sowie Intentionen des } \\
\text { Fahrroboters, der Verkehrsteilnehmer und weiterer Elemente in direkter } \\
\text { Fahrzeugumgebung } \\
\text { - Ziel: Kollisionsvermeidung im Quer- und Längsverkehr mit einem oder } \\
\text { mehreren Fahrzeugen der direkten Nachbarschaft entsprechend bereits } \\
\text { existierender V2X-Konzepte }\end{array}$ \\
\hline $\begin{array}{l}\text { Bereitstellen } \\
\text { von Umwelt- } \\
\text { informationen }\end{array}$ & $\begin{array}{l}\text { - Teilen von Informationen über die Fahrzeugumwelt, die vom Fahrroboter } \\
\text { wahrgenommen wurden, mit den Verkehrsteilnehmern sowie einer Ver- } \\
\text { kehrszentrale in direkter Umgebung } \\
\text { - Ziel: Bedatung einer optimierten Karte als Informationsquelle für Positio- } \\
\text { nierung, Gefahrenerkennung, Navigation etc. }\end{array}$ \\
\hline $\begin{array}{l}\text { Aktualisierung } \\
\text { der Fahrroboter- } \\
\text { fähigkeit }\end{array}$ & $\begin{array}{l}\text { - Update des Herstellers, das die (Fahr-)Fähigkeiten des Fahrroboters } \\
\text { verbessert }\end{array}$ \\
\hline $\begin{array}{l}\text { Fahrroboter- } \\
\text { überwachung }\end{array}$ & $\begin{array}{l}\text { - Teilen von Informationen über den Zustand, die Fähigkeiten und } \\
\text { die Intentionen des Fahrroboters mit berechtigten Instanzen } \\
\text { - Ziel: Sicherung von Beweismaterial (Event Data Recording) für die } \\
\text { Rekonstruktion eines Unfallhergangs und Mitteilung von Fehlfunktionen } \\
\text { oder Gefahrensituationen durch Selbstdiagnose an den Hersteller }\end{array}$ \\
\hline $\begin{array}{l}\text { Insassen- } \\
\text { überwachung }\end{array}$ & $\begin{array}{l}\text { - Teilen von Zustandsinformationen (Video, Audio, Herzschlag etc.) über } \\
\text { den Beförderten mit einer Notrufzentrale oder einem Dienstleister } \\
\text { - Weitergabe der Informationen an Berechtigte ohne Aktivität des Insassen } \\
\text { - Ziel: Überwachung der Gesundheit und Sicherheit des Beförderten }\end{array}$ \\
\hline Insassennotruf & $\begin{array}{l}\text { - aktive Kontaktierung von Notrufzentrale oder Dienstleister durch Insasse } \\
\text { im Notfall } \\
\text { - aktive Entscheidung des Insassen, ob er Informationen teilt oder nicht }\end{array}$ \\
\hline
\end{tabular}

\subsubsection{Merkmal G: Verfügbarkeitskonzept}

\subsubsection{Motivation}

Im Normalbetrieb wird das Fahrzeug durch den Fahrroboter innerhalb des zugelassenen Einsatzbereichs autonom bewegt. Erkennt der Fahrroboter eine i.A. nicht vorhersehbare Funktionsgrenze, übergibt der Fahrroboter an ein spezifiziertes Verfügbarkeitskonzept, welches festlegt, wie die Fahrmission fortgesetzt wird. Solche Funktionsgrenzen können beispielsweise durch unbekannte Hindernisse auf der Fahrbahn auftreten, die eine Weiterfahrt innerhalb der Entscheidungsautonomie nicht mehr zulassen. Ein Beispiel für ein solches Hindernis ist ein Ast, der so auf die Fahrbahn ragt, dass das Fahrzeug den Ast berühren muss, um die Fahrt fortzusetzen. In welchem Umfang das Verfügbarkeitskonzept 
die komplette Fahraufgabe übernimmt oder dem Fahrroboter nur eine Entscheidung abnimmt, wird bewusst offen gelassen.

\subsubsection{Merkmalsausprägungen}

1. keine Verfügbarkeitsergänzung

2. Verfügbarkeitsfahrer

3. teleoperiertes Fahren

4. Lotsen-Service

5. elektrisches Abschleppen

In Tab. 2.8 werden die unterschiedlichen Verfügbarkeitskonzepte näher erläutert. Die Übergabe vom Fahrroboter auf das alternative Verfügbarkeitskonzept ist risikominimal umzusetzen. Der Fahrroboter überführt das Fahrzeug für die Übergabe in den risikominimalen Zustand, der für eine Übergabe an das jeweilige Verfügbarkeitskonzept geeignet ist. Die entsprechenden Schnittstellen für den Verfügbarkeitsfahrer, die Fernsteuerung, einen Lotsen oder das Abschleppen müssen zur Verfügung stehen.

Tab. 2.8 Ausprägungen des Merkmals G

\begin{tabular}{|l|l|}
\hline Ausprägung & Beschreibung \\
\hline $\begin{array}{l}\text { keine Verfügbarkeits- } \\
\text { ergänzung }\end{array}$ & $\begin{array}{l}\text { Abwarten des Fahrroboters, bis durch äußere Einflüsse die Szene } \\
\text { wieder beherrschbar und durch die Spezifikationen des Fahrroboters } \\
\text { abgedeckt wird }\end{array}$ \\
\hline Verfügbarkeitsfahrer & $\begin{array}{l}\text { Unterstützung des Fahrroboters durch einen Insassen bei der Bewäl- } \\
\text { tigung der Szene (offen, ob durch Übernahme der Fahrfunktion oder } \\
\text { durch Manöverkommandos) }\end{array}$ \\
\hline teleoperiertes Fahren & $\begin{array}{l}\text { Unterstützung des Fahrroboters durch einen Dienstleister bei der } \\
\text { Bewältigung der Szene mithilfe einer Fernsteuerung }\end{array}$ \\
\hline Lotsen-Service & $\begin{array}{l}\text { Unterstützung des Fahrroboters durch eine besonders ausgebildete } \\
\text { Person bei der Bewältigung der Szene }\end{array}$ \\
\hline elektrisches & $\begin{array}{l}\text { Voraussetzung: für Regelungsaufgabe notwendige Hardware funk- } \\
\text { tionsfähig } \\
\text { Abschleppen }\end{array}$ \\
$\begin{array}{l}\text { Ansteuerung der Hardware durch Abschleppwagen mittels direkter } \\
\text { Verbindung }\end{array}$
\end{tabular}

\subsubsection{Merkmal H: Erweiterungskonzept}

\subsubsection{Motivation}

Mithilfe des autonomen Fahrens werden nicht zwangsläufig, vor allem nicht zu Beginn der Einführung, alle Einsatzbereiche abgedeckt, die für die Bewältigung aller Transportaufgaben benötigt werden. Teilbereiche verbleiben, die nicht autonom bewältigt werden 
können. Um dennoch die Mobilitätsbedürfnisse der Kunden zu erfüllen, könnten Bereiche außerhalb des Einsatzbereichs des autonomen Fahrens mithilfe von Erweiterungskonzepten abgedeckt werden. Das Erweiterungskonzept beschreibt, ob und womit es ermöglicht wird, die Fahrzeugführung außerhalb des für autonomes Fahren spezifizierten Einsatzbereichs auszuführen.

\subsubsection{Merkmalsausprägungen}

\section{1. keine Erweiterung}

2. Fahrer

3. teleoperiertes Fahren

4. Lotsen-Service

5. Extra-Transportmittel

In Tab. 2.9 werden den einzelnen Konzepten Beschreibungen detaillierter Charakteristiken zugewiesen. Wenn auf das Erweiterungskonzept Fahrer gesetzt wird, bedingt dies, dass eine Fahrzeugführungsschnittstelle („,Fahrerarbeitsplatz“) zur Verfügung steht. Außerdem wird vorausgesetzt, dass eine fahrtüchtige und autorisierte Person als Insasse die Fahrt außerhalb des Einsatzbereichs des autonomen Fahrens begleitet. Für die anderen denkbaren, aus heutiger Sicht noch futuristisch wirkenden Ausprägungen (teleoperiertes Fahren sowie Lotsen-Service) ist ein für diese Variante notwendiger Dienst bzw. eine Schnittstelle bereitzustellen.

Tab. 2.9 Ausprägungen des Merkmals H

\begin{tabular}{|c|c|}
\hline Ausprägung & Beschreibung \\
\hline keine Erweiterung & $\begin{array}{l}\text { - der Einsatzbereich wird nicht erweitert } \\
\text { - vollständige Abdeckung eines Einsatzbereichs für spezifizierte } \\
\text { Transportaufgaben: exklusiv-autonomes Fahrzeug } \\
\text { - bei Überdeckung des Einsatzbereichs mit dem von aktuellen Fahr- } \\
\text { zeugen: vollautonomes Fahrzeug }\end{array}$ \\
\hline Fahrer & - Übernahme der Fahraufgabe durch einen Menschen \\
\hline teleoperiertes Fahren & - Übernahme der Fahraufgabe durch einen externen Operator \\
\hline Lotsen-Service & - siehe Tab. 2.8 \\
\hline Extra-Transportmittel & $\begin{array}{l}\text { - Überführen des Fahrroboters an den Grenzen des Einsatzbereichs } \\
\text { zu einem Extra-Transportmittel, sodass dieses Transportmittel die } \\
\text { Transportaufgabe weiter ausführt } \\
\text { - Beispiel: Langstreckentransport von Stadtfahrzeugen mithilfe eines } \\
\text { „Autozugs“ oder mithilfe eines Konzepts ähnlich einer elektronischen } \\
\text { Deichsel }\end{array}$ \\
\hline
\end{tabular}




\subsubsection{Merkmal I: Eingriffsmöglichkeiten}

Nach Donges [3] müssen die drei primären Fahraufgaben Navigation, Bahnführung und Stabilisierung erfüllt werden, um ein Fahrzeug zu einem Fahrtziel zu führen. Die Stabilisierungsaufgabe wird u. a. bei Löper [1] durch die Regelungsaufgabe („Control Level“) ersetzt: Die Regelungsaufgabe beinhaltet die Stabilisierung, und darüber hinaus besitzt sie die Möglichkeit, das Fahrzeug in einen fahrdynamisch instabilen Zustand zu überführen, mit dem Ziel, die Fahraufgabe zu erfüllen. Somit werden im Folgenden die für den Menschen relevanten Fahraufgaben Navigation, Bahnführung und Regelung betrachtet.

Diese Fahraufgaben werden nach der Definition des vollautomatisierten Fahrens komplett dem Fahrroboter übergeben: Bekommt der Fahrroboter ein Fahrtziel vorgegeben, erfüllt dieser die Navigations-, Bahnführungs- und Regelungsaufgabe und führt das Fahrzeug zu dem Fahrtziel. Zwar muss der Fahrroboter diese Fahraufgaben erfüllen, der Aufbau der Architektur des Fahrroboters ist davon jedoch zunächst unabhängig. Im Gegensatz dazu werden aktuelle Serienfahrzeuge, mit Ausnahme von Gefahrensituationen (ABS, ESC, AEB), durch den menschlichen Fahrer geführt. Dem Menschen wird aktuell immer die Möglichkeit geboten, Assistenzsysteme durch die Betätigung der Stellelemente an seinem Fahrerarbeitsplatz zu korrigieren oder zu übersteuern. Somit existieren zwei Instanzen - der Insasse sowie der Fahrroboter - die prinzipiell die Fähigkeiten besitzen, ein Fahrzeug zu führen.

Zusätzlich existieren Ideen und Konzepte der Fahrzeugfernsteuerung (Tele-Operation), bei denen fahrzeugexterne Instanzen in die Fahrzeugführung eingreifen. Existieren eine Kommunikation und eine entsprechende Schnittstelle für die Fahrzeugaußenwelt, dann besitzen diese externen Instanzen ebenfalls die Möglichkeit, auf die Fahrzeugführung Einfluss zu nehmen. Somit können insgesamt die drei Instanzgruppen Intern, Fahrzeug und Extern die Fahrzeugführung übernehmen.

Für die vereinfachte Beschreibung des Merkmals sind die Insassen (volljährig, minderjährig, Menschen mit einschränkender Behinderung etc.) zur Gruppe Intern und die sich außerhalb des Fahrzeugs befindenden Einflussgrößen (hoheitliche Autoritäten (z. B. Polizei), Fahrzeughalter (wenn nicht Teil der Gruppe Intern), Bevollmächtigter etc.) zur Gruppe Extern zusammengefasst. Werden die Instanzen unabhängig voneinander betrachtet, ergeben sich folgende Fragen bezüglich deren Eingriffsmöglichkeiten:

1. Auf welcher Fahrzeugführungsebene hat die Instanz die Möglichkeit einzugreifen?

2. Für welche Fahrzeugführungsebene hat die Instanz die Befugnis einzugreifen?

Die erste Frage wird durch das Fahrzeugkonzept des Use-Case beantwortet. Soll die Instanz die Möglichkeit haben einzugreifen, dann wird vorausgesetzt, dass eine geeignete Schnittstelle für diese Instanz im Fahrzeugkonzept vorgesehen ist. Die zweite Frage erfordert eine rechtliche Regelung, die definiert, welche Befugnisse für Instanzen entsprechend deren Eigenschaften und Zuständigkeiten vergeben werden. Wer diese Regeln erstellt und prüft, ob es eine Art Fahrprüfung für die unterschiedlichen Ebenen gibt und Autorisierungen wie Führerschein oder Zugangscodes benötigt werden, soll hier nicht weiter ausgeführt werden. 
Daraus folgen unterschiedliche Kombinationen zwischen Möglichkeiten des Eingriffs, die das Fahrzeugkonzept bereitstellt, und der Befugnis eines Eingriffs, die eine Instanz besitzt:

Tab. 2.10 Kombinationen von Möglichkeiten und Befugnis zur Übernahme der Fahrzeugführung

\begin{tabular}{|l|l|l|}
\hline Variante & $\begin{array}{l}\text { Möglichkeit des Eingriffs, vorge- } \\
\text { geben durch das Fahrzeugkonzept }\end{array}$ & $\begin{array}{l}\text { Befugnis zum Eingreifen, die die Instanz } \\
\text { besitzt }\end{array}$ \\
\hline a) & $\begin{array}{l}\text { auf allen drei Ebenen (Navigation, } \\
\text { Bahnführung und Regelung) }\end{array}$ & auf allen drei Ebenen \\
\hline b) & auf allen drei Ebenen & keine (z. B. Minderjähriger als Fahrzeugführer) \\
\hline c) & keine & $\begin{array}{l}\text { auf allen drei Ebenen (z. B. Fahrer auf Rück- } \\
\text { sitz, der nicht eingreifen kann) }\end{array}$ \\
\hline d) & auf einer bestimmten Ebene & auf einer anderen Ebene \\
\hline
\end{tabular}

Lediglich Variante a) führt dazu, dass der Fahrroboter durch die Instanz in einer der Ebenen der Fahraufgabe beeinflusst und/oder überstimmt werden kann.

Für die Beschreibung der Use-Cases folgt daraus, dass die Instanzen aufgelistet werden, bei denen mindestens eine Befugnis mit einer angebotenen Möglichkeit durch das Fahrzeugkonzept übereinstimmt.

Zusätzlich wird angenommen, dass durch eine rechtliche Regelung der Missbrauch geahndet und somit verhindert wird. Diese Annahme wird auch bei aktuellen Fahrzeugkonzepten getroffen, weshalb Kinder beispielsweise nicht durch die Technik daran gehindert werden, ein Fahrzeug zu führen, sondern durch eine entsprechende gesetzliche Regelung in Verbindung mit der Aufsichtspflicht.

Werden die Instanzen nun gleichzeitig betrachtet und können folglich die Instanzen gleichzeitig auf den drei Ebenen der Fahraufgabe wirken, stellt sich die dritte Frage:

3. Welche Instanz ist im Konfliktfall des gleichzeitigen Eingriffs dominant und wie ist die Rangordnung zwischen den Instanzen definiert? (s. Abb. 2.9)

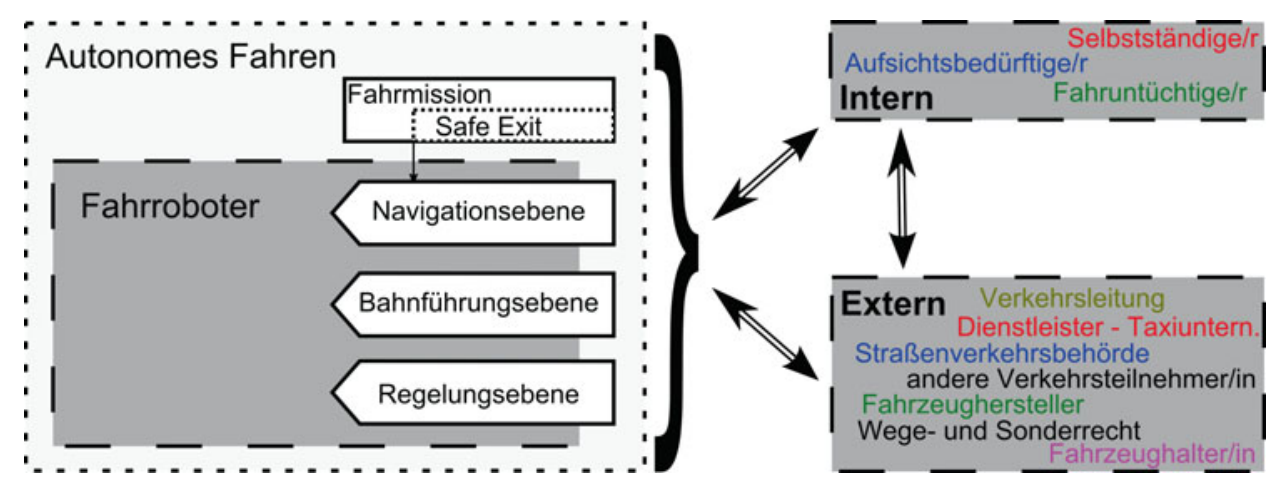

Abb. 2.9 Eingriffskonflikt zwischen Instanzen bei der Ausführung der Fahraufgabe 
Um diese Frage für die Beschreibung der Use-Cases zu beantworten, muss der Eingriff der Instanzen mit einer Priorität belegt werden. Welche Instanz dominiert die anderen und bestimmt damit das Fahrzeugverhalten auf den unterschiedlichen Ebenen der Fahraufgabe? Eine Rangordnung der Instanzen ist bei der Fahrzeugauslegung zu implementieren. Dabei ist zu beachten, dass zusätzlich zu der Rangordnung zwischen den Instanzen auch eine Rangordnung zwischen den Ebenen der Fahraufgabe existiert: Die Regelung überstimmt immer die Bahnführung und die Bahnführung immer die Navigationsebene; deshalb ist zusätzlich definiert, dass nur auf einer Ebene durch Intern oder Extern eingegriffen werden kann. Die Instanz mit der höchsten Priorität unterbindet die anderen Eingriffe.

Mithilfe des autonomen Fahrens besteht die Möglichkeit, dass ausschließlich Personen befördert werden, die nicht die Fähigkeit besitzen, die Fahraufgabe auszuführen oder die Fahrmission zu ändern. Um einem Insassen aber immer die Möglichkeit zu geben, auf schnellstem Weg sicher auszusteigen, ist der Safe-Exit als besondere Fahrmission eingeführt. Bekommt der Insasse auf den Safe-Exit Zugriff mit der höchsten Priorität, kann er zwar das Fahrtziel nicht unbedingt ändern, aber möglichst schnell das Fahrzeug verlassen.

\subsection{Grundlegende Definitionen}

Grundlegende Begriffe, die zur Beschreibung der Use-Cases verwendet wurden, sind im Folgenden definiert.

Assistiert: Automatisierungsgrad 1 nach Bast [1]: „Fahrer führt dauerhaft entweder die Quer- oder die Längsführung aus. Die jeweils andere Fahraufgabe wird in gewissen Grenzen vom System ausgeführt.

- Der Fahrer muss das System dauerhaft überwachen.

- Der Fahrer muss jederzeit zur vollständigen Übernahme der Fahrzeugführung bereit sein.“

Autonomes Fahren: Die Fahraufgabe nach Donges [3] wird „vollautomatisiert“ ausgeführt. Diese Definition wird erweitert um die Annahme, dass die Ausführung der Fahraufgabe auf Basis maschinell autonomen Verhaltens, innerhalb eines vorher festgelegten Verhaltensrahmens, geschieht.

Autonomes Fahrzeug: Das Fahrzeug ist mit einem Fahrroboter ausgestattet und besitzt deshalb die Möglichkeit, autonom zu fahren. Welche Bereiche dieses autonome Fahrzeug abdeckt, ist nicht definiert.

Bahnführen: Laut Donges besteht die Bahnführungsaufgabe „(...) im Wesentlichen darin, aus der vorausliegenden Verkehrssituation sowie aufgrund des geplanten Fahrtablaufs die als sinnvoll erachteten Führungsgrößen wie Sollspur und Sollgeschwindigkeit 
abzuleiten und antizipatorisch im Sinn einer Steuerung (open loop control) einzugreifen, um günstige Vorbedingungen für möglichst geringe Abweichungen zwischen Führungsund Istgrößen zu schaffen“. [3]

Driver-Only: Automatisierungsgrad 0 nach BASt [1]: „Fahrer führt dauerhaft (während der gesamten Fahrt) die Längsführung (Beschleunigen/Verzögern) und die Querführung (Lenken) aus."

Dynamische Elemente: Dynamische Elemente sind nach Geyer et al. [5] temporal oder spatial variable Elemente wie andere Verkehrsteilnehmer, Zustände von Lichtsignalanlagen, Licht- und Wetterbedingungen.

Einsatzbereich: Ein durch die Szenerie explizit und durch die erlaubte Geschwindigkeit implizit spezifizierter räumlicher und/oder zeitlicher Bereich, in dem das Fahrzeug autonom durch den Einsatz des Fahrroboters bewegt werden kann.

Einsatzgrenze $^{1}$ : Die Einsatzgrenze ist explizit durch die Szenerie und implizit z.B. durch eine Geschwindigkeit spezifiziert und somit eine vorhersehbare Grenzlinie, an der die Fahraufgabe übergeben wird.

Exklusiv-autonomes Fahrzeug (autonomous-only vehicle): Ein Fahrzeug, das alle Strecken, für die es als Fahrzeug spezifiziert ist, von Start bis Ziel autonom fährt. Diese Definition geht über die Unterteilung des Automatisierungsgrads des Fahrens (BASt [1]) hinaus und beschreibt das Fahrzeug an sich.

Fahrer: Der Fahrer ist der (geschlechtsneutral gemeinte) fahrzeugführende Mensch ohne weitere Spezifikation der Fahrfähigkeit, also innerhalb der Bandbreite der Menschen, die eine Fahrerlaubnis besitzen. Er ist das Subjekt der Autonomie bei nicht vollautomatisiertem Fahren.

Fahrmission: Die Fahrmission beschreibt die Fahrt vom Start zum Ziel als Ausführung einer Transportaufgabe.

Fahrroboter: Der Fahrroboter ist die Implementation der maschinellen (Fahr-)Fähigkeiten. Der Fahrroboter besteht aus Hardware-Komponenten (Sensoren, Prozessoren und Aktoren) und Software-Elementen. Er agiert als Hard- und Software analog zur Rolle des Fahrers in heutigen Fahrzeugen als Subjekt ${ }^{2}$. (Die Begriffsbildung für dieses System ist nicht abgeschlossen.)

Funktionsgrenze $^{3}$ : Eine im erlaubten Einsatzbereich auftretende, nicht im Detail vorhersehbare Bedingung, die einer Fortsetzung der autonomen Fahrt entgegensteht. Auch wenn die Grenze nicht vorhersehbar ist, erkennt sie der Fahrroboter dennoch frühzeitig.

Maschinelle (Fahr-)Fähigkeiten: Mit den maschinellen (Fahr-)Fähigkeiten sind die Fähigkeiten der Perzeption, Kognition, Verhaltensentscheidung und die Verhaltensausführung gemeint.

1 Die Einsatzgrenze entspricht der Systemgrenze Kategorie 1 der BASt-Betrachtung [1].

2 ,A system which is capable of taking decisions depending on sensor data processed internally has additional degrees of freedom as compared to one with direct sensor data to actuator feedback or one without any capability of control actuation. The former one is termed a 'subject', the last one an 'object' (...)" [4]

3 Die Funktionsgrenze entspricht der Systemgrenze Kategorie 2 der BASt-Betrachtung [1]. 
Navigieren: Nach Donges umfasst das Navigieren ,(...) die Auswahl einer geeigneten Fahrtroute aus dem zur Verfügung stehenden Straßennetz sowie eine Abschätzung des voraussichtlichen Zeitbedarfs. Wenn Informationen über aktuelle Störeinflüsse wie z. B. Unfälle, Baustellen oder Verkehrsstauungen vorliegen, kann eine veränderte Routenplanung erforderlich werden." [3]

Safe-Exit: Der Safe-Exit ist eine besondere Fahrmission. Diese überführt das Fahrzeug auf schnellstem Weg in einen Zustand, der es dem Insassen ermöglicht, das Fahrzeug sicher zu verlassen.

Situation: Eine eindeutige Definition des Begriffs Situation für die Use-Case-Beschreibung steht noch aus. Insbesondere ist zwischen einer „objektiven, allwissenden Situation(-sbeschreibung)“ und einer „subjektiven, projektiven Situation(-sbeschreibung)“ zu unterscheiden.

Stabilisieren: Als Erfüllung der Stabilisierungsaufgabe hat nach Donges „,...) der Fahrer durch entsprechende korrigierende Stelleingriffe dafür zu sorgen, dass im geschlossenen Regelkreis (closed loop control) die Regelabweichungen stabilisiert und auf ein für den Fahrer annehmbares Maß kompensiert werden." [3]

Szene: Die Szene, definiert nach Geyer et al. [5], besteht aus der Szenerie, dynamischen Elementen und optionalen Fahranweisungen. Eine Szene startet entweder mit dem Ende der vorherigen Szene oder -im Fall der ersten Szene - mit einer definierten Startszene. In einer Szene sind alle Elemente, deren Verhalten und die Position des Ego-Fahrzeugs definiert. Die dynamischen Elemente ändern in einer Szene ihren Zustand.

Szenerie: Unter dem Begriff der Szenerie nach Geyer et al. [5] wird die statische Umgebung des Fahrzeugs verstanden. Damit sind u. a. die Geometrie von vordefinierten Straßentypen, die Anzahl an Fahrstreifen, der Straßenverlauf, die Position von Verkehrszeichen und Lichtsignalanlagen sowie weitere statische Objekte, wie z.B. Baustelleneinrichtungen sowie natürliche (z. B. Sträucher und Bäume) oder künstliche Gebilde (z. B. Häuser, Wände) gemeint.

Transportaufgabe: Die Transportaufgabe beschreibt den Transport eines definierten Transportgegenstandes (Fahrzeug, Ladegut, Passagier usw.) von einem Startort zu einem Zielort. Beispiele für Transportaufgaben sind ein Fahrzeug zu parken oder einen Passagier zu einem gewünschten Zielort zu bringen.

Vollautomatisiert: Automatisierungsgrad 4 nach Bast [1]: „Das System übernimmt Quer- und Längsführung vollständig in einem definierten Anwendungsfall.

- Der Fahrer muss das System dabei nicht überwachen.

- Vor dem Verlassen des Anwendungsfalles fordert das System den Fahrer mit ausreichender Zeitreserve zur Übernahme der Fahraufgabe auf.

- Erfolgt dies nicht, wird in den risikominimalen Systemzustand zurückgeführt.

Systemgrenzen werden alle vom System erkannt, das System ist in allen Situationen in der Lage, in den risikominimalen Systemzustand zurückzuführen." 
Vollautonomes Fahrzeug: Ein solches Fahrzeug kann auf gleichem Niveau wie „Driveronly“-Fahrzeuge nahezu alle Strecken autonom fahren. Diese Definition geht über die Unterteilung des Automatisierungsgrads des Fahrens (BASt [1]) hinaus und beschreibt das Fahrzeug an sich.

\section{Literatur}

1. Gasser, T.M., Arzt, C., Ayoubi, M., Bartels, A., Bürkle, L., Eier, J., Flemisch, F., Häcker, D., Hesse, T., Huber, W., Lotz, C., Maurer, M., Ruth-Schumacher, S., Schwarz, J., Vogt, W.: Rechtsfolgen zunehmender Fahrzeugautomatisierung. Gemeinsamer Schlussbericht der Projektgruppe. Berichte der Bundesanstalt für Strassenwesen - Fahrzeugtechnik (F), vol. 83. Wirtschaftsverl. NW Verl. für neue Wissenschaft, Bremerhaven, 2012

2. Anlehnung an das Autonomieverständnisses nach Kant interpretiert durch Feil, E.: Autonomie und Heteronomie nach Kant. Zur Klärung einer signifikanten Fehlinterpretation. In: Freiburger Zeitschrift für Philosophie und Theologie, 29/1-3, 1982, S. 389-441 (abgedruckt in Feil, E. Antithetik neuzeitlicher Vernunft. „Autonomie - Heteronomie“ und „rational - irrational“, Göttingen 1, Teil I, S. 25-112)

3. Donges, E.: Fahrerverhaltensmodelle. In: Winner, H. et al.: Handbuch Fahrerassistenzsysteme. 2. Auflage, S. 15-23, Vieweg+Teubner Verlag, Wiesbaden, 2012

4. Dickmanns, E.D.: Subject-object discrimination in 4D dynamic scene interpretation for machine vision. In: [1989] Proceedings. Workshop on Visual Motion, Irvine, CA, USA, 20-22 March 1989, pp. 298-304. doi: 10.1109/WVM.1989.47122

5. Geyer, S.; Baltzer, M.; Franz, B.; Hakuli, S.; Kauer, M.; Kienle, M.; Meier, S.; Weißgerber, T.; Bengler, K.; Bruder, R.; Flemisch, F. O.; Winner, H.: Concept and Development of a Unified Ontology for Generating Test and Use-Case Catalogues for Assisted and Automated Vehicle Guidance. IET Intelligent Transport Systems. Zur Veröffentlichung angenommen. 2013

6. Kempen, B.: Fahrerassistenz und Wiener Weltabkommen. In: 3. Sachverständigentag von TÜV und DEKRA: Mehr Sicherheit durch moderne Technologien, 25./26. Februar 2008 in Berlin

7. Kategorien der Verkehrswege für den Kfz-Verkehr (3.4.1) aus den Richtlinien für integrierte Netzgestaltung Ausgabe 2008

8. Löper, C.; Flemisch, F.: Ein Baustein für hochautomatisiertes Fahren: Kooperative, manöverbasierte Automation in den Projekten H-Mode und HAVEit. 6. Workshop Fahrerassistenzsysteme in Hößlinsülz, 2009 